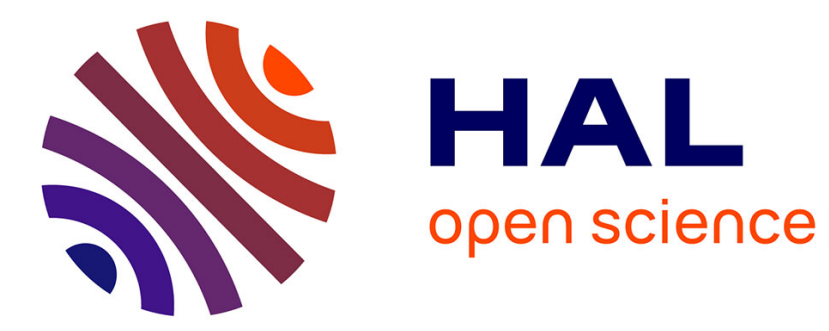

\title{
Nonlinear control of parallel manipulators for very high accelerations without velocity measurement: stability analysis and experiments on Par2 parallel manipulator
}

Guilherme Sartori Natal, Ahmed Chemori, François Pierrot

\section{- To cite this version:}

Guilherme Sartori Natal, Ahmed Chemori, François Pierrot. Nonlinear control of parallel manipulators for very high accelerations without velocity measurement: stability analysis and experiments on Par2 parallel manipulator. Robotica, 2016, 34 (01), pp.43-70. 10.1017/S0263574714001246 . lirmm01342856

\author{
HAL Id: lirmm-01342856 \\ https://hal-lirmm.ccsd.cnrs.fr/lirmm-01342856
}

Submitted on 10 Sep 2019

HAL is a multi-disciplinary open access archive for the deposit and dissemination of scientific research documents, whether they are published or not. The documents may come from teaching and research institutions in France or abroad, or from public or private research centers.
L'archive ouverte pluridisciplinaire HAL, est destinée au dépôt et à la diffusion de documents scientifiques de niveau recherche, publiés ou non, émanant des établissements d'enseignement et de recherche français ou étrangers, des laboratoires publics ou privés. 


\title{
Nonlinear Control of Parallel Manipulators for Very High Accelerations Without Velocity Measurement: Stability Analysis and Experiments on Par2 Parallel Manipulator
}

\author{
Guilherme Sartori-Natal \\ KU Leuven, Department of Mechanical Engineering, Celestijnenlaan 300B \\ BE-3001 Heverlee, Belgium. (Guilherme.SartoriNatal@mech.kuleuven.be) \\ Ahmed Chemori and François Pierrot \\ LIRMM, Univ. Montpellier 2 - CNRS, UMR 5506 - CC 477, 161 rue Ada \\ 34095 Montpellier, France. (chemori,pierrot)@lirmm.fr
}

\begin{abstract}
This paper presents a comparison between control/state estimation methods applied on Par2 parallel manipulator for pick-and-place applications, as well as a discussion about the mechanical vibrations issue that may become important when reaching very high accelerations. Real-time experiments were performed firstly to compare two controllers (a linear Proportional-Derivative (PD) controller and a nonlinear/adaptive Dual Mode (DM) controller) complied with the same High-gain observer (HGO) in order to estimate the articular velocities, and secondly to compare three state observers (a Lead-lag (LL) based, an Alpha-beta-gamma (ABG) and a High-gain observer) complied with the same nonlinear DM controller. The stability analysis of the Par2 robot under the control of the proposed Dual Mode controller (complied with the High-gain observer for joint velocity estimation) is also provided. Some small mechanical vibrations were noticed when reaching $20 \mathrm{G}$ of acceleration, which means that it can become an important issue for higher accelerations. Some suggestions are then made for future investigations, in order to avoid/damp these vibrations.
\end{abstract}


Keywords: Parallel manipulators, Nonlinear control, Adaptive control, State observers, Pick-and-place.

It is usually mentioned that parallel robots have attracted a considerable attention on the few past decades. It must be mentioned, however, that the first design patent of a parallel robot (a motion platform for the entertainment industry) was applied by J. E. Gwinnett in 1928, being issued in 1931 [1]. The first industrial parallel robot (for automated spray painting) to be built was patented by W. L. V. Pollard Jr. in 1942. A few years later (more precisely in 1947), the parallel robot that became the most popular in the industry of that time (the variable-length-strut octahedral hexapod for tire testing) was invented by E. Gough. During the 60's, it was K. Cappel who independently designed the very same hexapod, had it patented and licensed to the first flight simulator companies, making it the first commercial octahedral hexapod motion simulator. Yet, it was D. Stewart who, unintentionally, made Gough's concept popular and proposed, once again, this idea for flight simulators, machine tools and universal milling machines [1].

In the early 80's, a new type of parallel robots was invented by R. Clavel, namely the Delta robot [2]. Its basic idea consisted in the use of parallelograms, which allows an output link to remain at a fixed orientation with respect to an input link.

Since then, several robots with the same concept have been designed, and its applications vary from the packaging/pharmaceutical industrial domain (pick-andplace/assembly tasks), the medical domain (i.e. carry a $20 \mathrm{~kg}$ microscope for surgery purposes) and even the entertainment domain (haptic game controllers).

The main advantages of parallel robots in comparison to their serial counterparts are their higher stiffness and lighter structures, which allow them to achieve very 
high accelerations/velocities. In order to achieve such accelerations and perform an accurate movement, a good controller must be used. By using simple linear singleaxis controllers (such as a Proportional Derivative (PD) controller), the tracking performance can be limited, especially when the robot has highly nonlinear dynamics and/or when the velocities/accelerations are high [3]. In this case, a more advanced (nonlinear/adaptive) controller is necessary.

Different nonlinear/adaptive controllers have already been used for the control of parallel robots. In [3], a nonlinear adaptive feedforward controller was proposed for the control of the Hexaglide (a 6 dof parallel robot), in addiction to a PD feedback term. The main objective of this work was to show the convergence of the adaptive parameters in simulation. In [4], a control scheme similar to the so called computed torque controller ${ }^{1}$ [5] was also proposed to control the Hexaglide robot, but with experimental results that showed a good improvement in the trajectory tracking with respect to a $\mathrm{PD}$ controller, although no control signal has been presented. ***An adaptive fuzzy integral sliding mode controller has been proposed for parallel manipulators in [6]. This method was shown to provide good trajectory tracking performances and robustness without the undesired chattering effect of standard sliding mode controllers. However, as only simulation results were provided, the real-time experimental applicability of such control approach is still to be verified. The importance of adequate identification of the parameters of parallel robots was also emphasized in [7], [8].***

It is well known that in most control algorithms it is assumed that the joint velocities are available, that is, they need to be calculated or estimated. The easiest way

\footnotetext{
${ }^{1}$ With the modification that the dynamic model is computed from the desired values, instead of the actual joint coordinates.
} 
to compute the articular velocities consists in a straight numerical derivative of the measured articular positions. However, if the measured positions are noisy or do not have a good enough resolution, this technique will amplify the noise/quantization effect. In order to overcome this problem, an estimation of the articular velocities by means of observer-based techniques must be considered. The choice of the estimation mechanism is strongly influenced by the existence of uncertainty in the system model. Whereas model-based observers are usually restricted to cases where the model is exactly known, filters can provide a model-independent means of estimating velocity [9].

In this paper, two comparisons have been made. Firstly, a comparison between the trajectory tracking performance obtained by using two different controllers (a linear PD and a nonlinear/adaptive Dual Mode (originally referred as 'binary' in [10]) controller) complied with the same velocity estimator (High-gain observer), and then a comparison between three velocity estimators (Lead-lag based, Highgain and Alpha-beta-gamma observers) complied with the same nonlinear/adaptive Dual Mode controller. Another contribution of this paper consists in the stability analysis of the Par2 robot when controlled by the proposed Dual Mode controller, while having the High-gain observer as velocity estimator.

It is also important to emphasize that even with a good controller and a good state observer, it is very difficult to avoid mechanical vibrations for very high accelerations, which may cause loss of movements' precision. Therefore, a list of possible solutions to avoid/damp these vibrations is presented and discussed.

This paper is organized as follows. In section 1, the Par2 parallel robot is described. Section 2 presents the general dynamics of the system. Section 3 introduces the two proposed control algorithms, and shows that both of them need the velocity 
measurements. The proposed observers are described in section 4 . The stability analysis of the Par2 parallel manipulator is presented and discussed in section 5 . In section 6 , there are two sub-sections: the first one presenting the experimental results obtained by the two proposed controllers complied with the High-gain observer, and the second one presenting the experimental results obtained by the Dual Mode controller complied with three proposed observers. In section 7, a discussion about the mechanical vibrations that were noticed for $20 \mathrm{G}$ of acceleration and about possible solutions to avoid serious issues for higher accelerations is made. A conclusion about the current results and eventual future prospects is presented in section 8.

\section{PAR2 PARALLEL MANIPULATOR}

\subsection{Description of the Par2 robot}

Par2 is a two-dof parallel manipulator, illustrated in Figure 1, with the following characteristics:

- the platform (6) is a rigid body,

- only the two inner arms (3) are actuated,

- the two other arms (4) are linked to the frame (1) through passive revolute joints,

- inner arms (3) and (4) are connected to (6) with pairs of rods (5) mounted on ball joints (7),

- the rotations of the arms (4) are coupled in order to guarantee planar motions along $\mathrm{x}$ and $\mathrm{z}$ axes. 

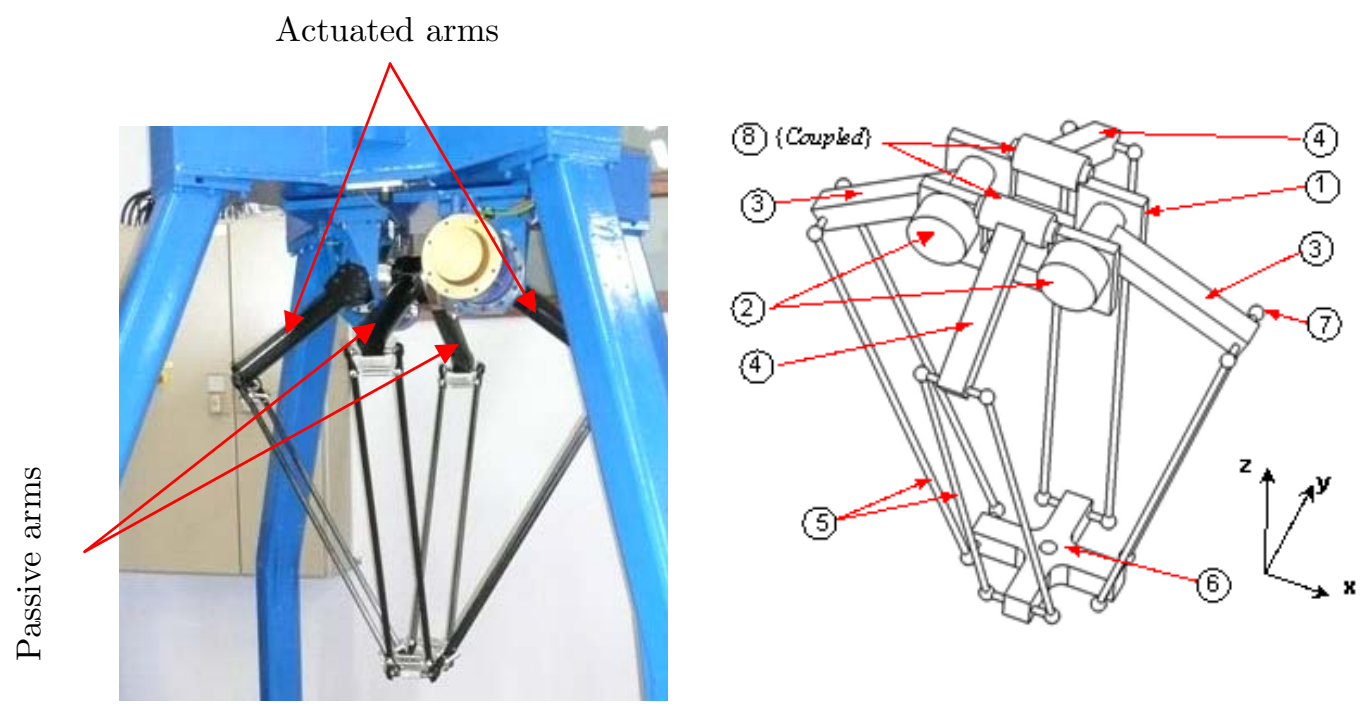

Figure 1: The two-dof parallel manipulator Par2: view of the robot (left), schematic view of its mechanical structure (right)

*** The geometric parameters of the robot are summarized in Table 1, where:

- $i$ : index of the kinematic chains $(\mathrm{i}=1,2)$,

- $l_{i}^{\text {arm }}$ : length of arms $P_{i} A_{i}$,

- $L_{i}^{\text {forearm }}$ : length of arms $A_{i} B_{i}$,

- $x_{p}$ : abscissa of the points $P_{i}$ defined by the parameters $(D+d)$,

- $d_{t p}$ : length of the traveling plate $B_{1} B_{2}$.

Table 1: Geometric Parameters of Par2

\begin{tabular}{|c|c|c|c|c|}
\hline$l_{i}[\mathrm{~m}]$ & $L_{i}[\mathrm{~m}]$ & $d_{t p}[\mathrm{~m}]$ & $D[\mathrm{~m}]$ & $z_{0}[\mathrm{~m}]$ \\
\hline 0.375 & 0.825 & 0.1 & 0.25 & 0.9 \\
\hline
\end{tabular}

$* * *$

The proposed architecture of the prototype has the following advantages [12]: 


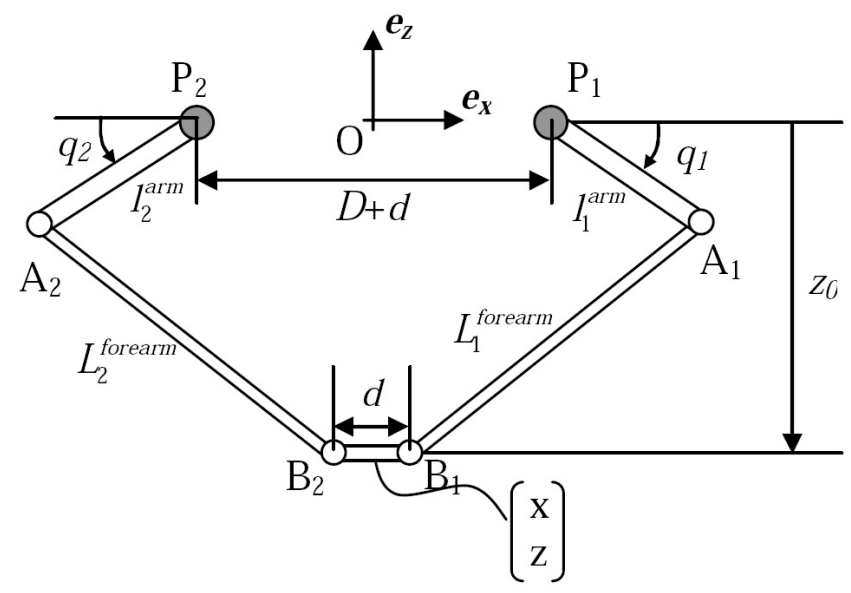

Figure 2: Geometric parameters of Par2: Side view [11]

- the two coupled passive chains made a constrained system supporting almost all the moments and force besides the driving forces,

- it can be designed with existing technologies and parts (can be made as light as for Delta [13] or Par4 [14] robots),

- it is symmetric with respect to motion plane and the yoz plane in its centered position.

The proper functioning of this two-dof parallel manipulator is guaranteed by the coupling of the rotation of arms (4). This constrains the platform of the robot to evolve in one plane. The coupling means that the rotation of the first arm in the clockwise direction involves the rotation of second one in the counterclockwise direction. For more details about the prototype Par2, the reader is referred to [12]. 


\section{GENERAL MANIPULATOR DYNAMICS}

The Lagrangian nonlinear dynamic model $[15,16]$ of robot manipulators that can make use of the control and observation tools proposed in this paper is described in its matrix form by the following equation:

$$
I(q) \ddot{q}+C(q, \dot{q}) \dot{q}+G(q)+f(q, \dot{q})=\tau
$$

where

$q^{T}=\left[q_{1}, q_{2}, \ldots, q_{n}\right] \in \mathbb{R}^{n}$ is the vector of articular positions,

$\dot{q}^{T}=\left[\dot{q}_{1}, \dot{q}_{2}, \ldots, \dot{q}_{n}\right] \in \mathbb{R}^{n}$ is the vector of articular velocities,

$\ddot{q}^{T}=\left[\ddot{q}_{1}, \ddot{q}_{2}, \ldots, \ddot{q}_{n}\right] \in \mathbb{R}^{n}$ is the vector of articular accelerations,

$I(q) \in \mathbb{R}^{n \times n}$ is the inertia matrix,

$C(q, \dot{q}) \in \mathbb{R}^{n \times n}$ is the matrix of Coriolis and centrifugal terms,

$G(q) \in \mathbb{R}^{n}$ is the vector of gravitational forces,

$f(q, \dot{q}) \in \mathbb{R}^{n}$ is the vector of friction forces,

$\tau \in \mathbb{R}^{n}$ is the vector of control inputs (torques generated by the actuators).

By considering that the dry friction has been neglected, the nonlinear dynamics of Par2 is given as follows:

$I_{\text {tot }}\left[\begin{array}{c}\ddot{q}_{1} \\ \ddot{q}_{2}\end{array}\right]=\tau+\frac{g}{2}\left(M_{\text {arm }}+M_{\text {forearm }}\right)\left[\begin{array}{c}l_{1} \cos \left(q_{1}\right) \\ l_{2} \cos \left(q_{2}\right)\end{array}\right]-J_{m}^{T} M_{\text {tot }}\left(\dot{J}_{m} \dot{q}-\left[\begin{array}{c}0 \\ g\end{array}\right]\right)-f_{v}\left[\begin{array}{c}\dot{q}_{1} \\ \dot{q}_{2}\end{array}\right]$ 
where $I_{t o t}=I_{m o t}+I_{a r m}+J_{m}^{T} M_{t o t} J$, being $I_{m o t}$ the motor driver inertia, $I_{\text {arm }}$ the arm inertia, $M_{t o t}=M_{t p}+n \frac{M_{\text {forearm }}}{2}$, being $M_{t p}$ the mass of the traveling plate (including the payload), $J$ is the Jacobian matrix and $\dot{J}$ its first time derivative, $g$ is the gravitational acceleration, $f_{v}$ is the viscous friction coefficient, $M_{\text {arm }}$ and $M_{\text {forearm }}$ are the masses of the arms and forearms, respectively, $l_{1}$ and $l_{2}$ are the lengths of the arms $\left(l_{1}=l_{2}\right)$ and $L_{1}$ and $L_{2}$ are the lengths of the forearms $\left(L_{1}=L_{2}\right)$. The dynamic parameters are presented in Table 2 .

Table 2: Dynamic Parameters of Par2

\begin{tabular}{|c|c|c|c|c|c|}
\hline$I_{\text {mot }}\left[\mathrm{kg} \cdot \mathrm{m}^{2}\right]$ & $I_{\text {arm }}\left[\mathrm{kg} \cdot \mathrm{m}^{2}\right]$ & $M_{\text {arm }}[\mathrm{kg}]$ & $M_{\text {forearm }}\left[\mathrm{kg} \cdot \mathrm{m}^{2}\right]$ & $M_{t p}[\mathrm{~kg}]$ & $f_{v}[$ N.m.s $]$ \\
\hline 0.19 & 0.019 & 0.82 & 0.14 & 0.5 & 0.8 \\
\hline
\end{tabular}

\section{PROPOSED CONTROL SCHEMES: A PD CONTROLLER AND A NONLINEAR/ADAPTIVE DUAL MODE CONTROLLER}

In order to implement both controllers, the desired articular trajectory (denoted by $\left.q_{d}(t)\right)$ must be provided. This trajectory is supposed uniformly bounded, twice continuously differentiable with its two first derivatives $\dot{q}_{d}(t)$ and $\ddot{q}_{d}(t)$ also uniformly bounded. In order to obtain this trajectory, the Cerebellum Path Generator [17] is used. It generates the desired *task space (which, in the present work, is equivalent to the Cartesian space)* trajectories that will be the input for the direct geometric model of the robot [12]. This scheme is presented in the diagram of Figure 3:

\subsection{Proportional-derivative controller}

As widely known, the Proportional-Derivative (PD) controller consists in the sum of a proportional and a derivative term, as follows: 


\begin{tabular}{|c|c|c|c|c|}
\hline Cerebellum & $\begin{array}{l}\text { Desired } \\
\text { Cartesian } \\
\text { Trajectory }\end{array}$ & Par2 & $\begin{array}{c}\text { Desired } \\
\text { Articular } \\
\text { Trajectory }\end{array}$ & $\frac{\mathbf{q}_{\mathrm{d}}}{\dot{\mathbf{q}}_{\mathrm{d}}}$ \\
\hline $\begin{array}{c}\text { PATH } \\
\text { GENERATOR }\end{array}$ & & $\begin{array}{l}\text { GEOMETRIC } \\
\text { MODEL }\end{array}$ & & $\ddot{q}_{d}$ \\
\hline
\end{tabular}

Figure 3: Details of the desired trajectories generation

$$
\tau=K_{p} e_{j}+K_{d} \dot{e}_{j}
$$

being $\tau$ the torques applied by the actuators (motors) of the robot, $K_{p}, K_{d}$ the proportional and derivative feedback gains, respectively, and

$$
e_{j}=q_{d}-q, \dot{e}_{j}=\dot{q}_{d}-\dot{q}
$$

where $e_{j}$ and $\dot{e}_{j}$ represent the joint position and velocity errors, $q_{d}$ and $\dot{q}_{d}$ represent the desired joint position and velocity and $q$ and $\dot{q}$ represent the actual joint position and velocity.

\subsection{NL/adaptive Dual Mode (DM) controller in the joint space}

The Dual Mode controller consists in the original one of [18] with the inclusion of a parametric projection law and the addition of a smooth saturation term which was proposed only for disturbance compensation purposes, in order to improve the robustness of the controlled system. The idea is to use a high adaptation gain together with a projection of the estimated parameters such that, to large tracking errors in the transient stage, the controller behaves approximately as a sliding mode controller, generating an exponential convergence to a residual domain arbitrarily 
small, and to smaller errors, it behaves as a parametric adaptation law. Other important advantages of the adaptation law in dual mode with respect to other adaptation laws or known robust control algorithms are listed below:

- Generation of continuous control signals,

- Limitation of the values of the estimated parameters thanks to a projection, which has the effect of reducing the effective gain of the controller when the tracking error increases (reducing the sensitivity to measurement noises).

According to [18], the following errors in the joint space are introduced:

$$
s=\dot{e}_{j}+\lambda e_{j}, \dot{q}_{r}=\dot{q}_{d}+\lambda e_{j}
$$

where $s$ is an auxiliary error, $\lambda$ is a positive constant, and $\dot{q}_{r}$ is the denominated "reference velocity" [18],[19]. The control architecture is illustrated in Figure 4, already considering the utilization of a state observer in order to estimate the joint velocities (which are not available in this case), with $\dot{e}_{j}$ substituted by $\hat{\dot{e}}_{j}$.

As illustrated in Figure 4, the control law consists in the sum of three terms: an "adaptive term", a "smooth variable structure term" and a "stabilizing term" $(K)$. The control input is given by:

$$
\tau=Y \hat{a}+\bar{d} S a t(\alpha s)+K s
$$

where

$$
\operatorname{Sat}(\alpha s)=\frac{\alpha s}{\|\alpha s\|+1}
$$




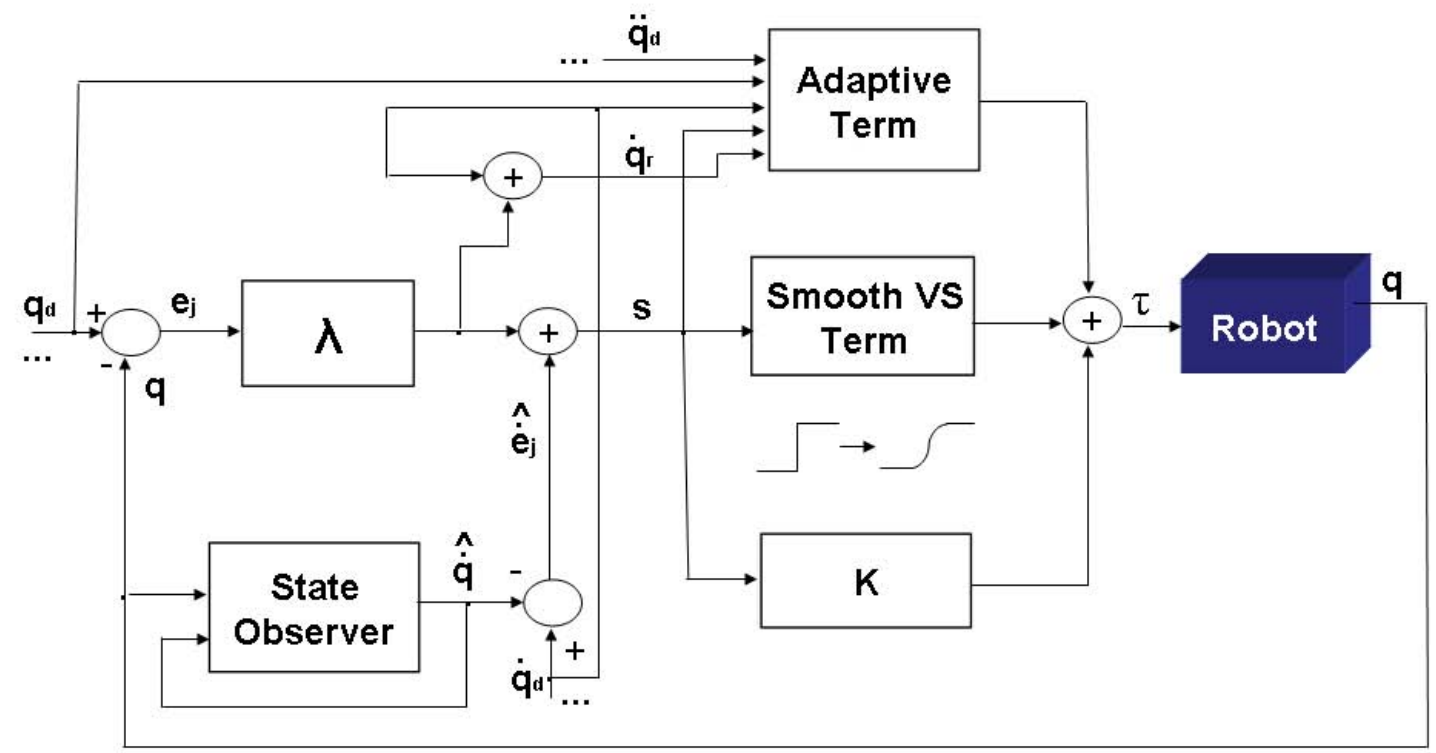

Figure 4: Block diagram of the Dual Mode controller

being $\bar{d}$ and $\alpha$ positive constants and $K$ a symmetric positive definite matrix. The function $\operatorname{Sat}($.$) is continuous with respect to its argument (with continuous partial$ derivatives and components limited to the interval $[-1,+1]$ ), and from Figure 5, it is possible to notice that the higher the chosen $\alpha$, the closer this function will be to a $\operatorname{sign}($.$) function.$

The vector $\hat{a}$ represents an estimate of the unknown parameters of the system given by the vector $a_{n o m}$, and $Y$ is the regressor vector (based on the dynamic model of the system). The adaptation of these estimated parameters is given by:

$$
\dot{\hat{\theta}}=-\gamma \operatorname{Proj}\left(s^{T} Y, \hat{\theta}\right)=-\sigma \hat{\theta}-\gamma s^{T} Y
$$

where 


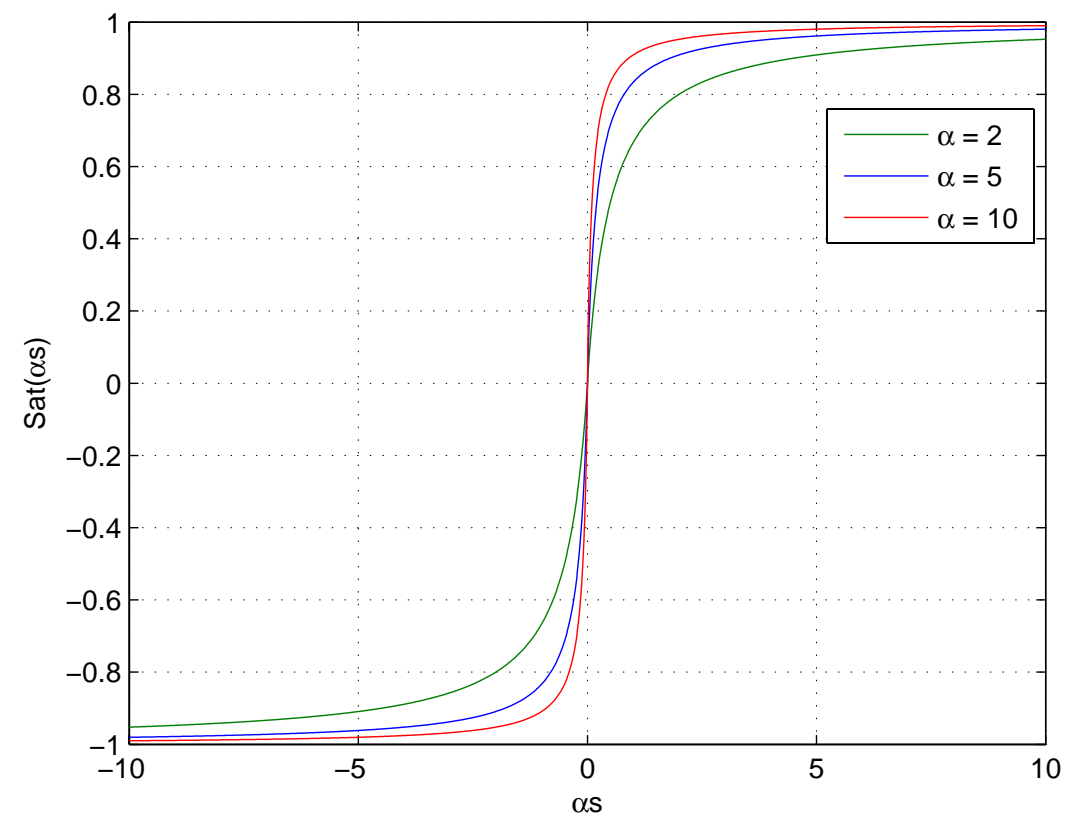

Figure 5: The effect of the parameter $\alpha$ on the smooth variable structure term

$$
\hat{\theta}=\hat{a}-a_{\text {nom }}
$$

is the difference between the currently estimated values and the nominal values of the parameters. The adaptation gain $\gamma$ is a real positive constant, and the variable $\sigma$ is adjusted as follows:

$$
\sigma=\left\{\begin{array}{cc}
0, \quad \text { if }\|\hat{\theta}\|<M_{\theta} \text { or } \sigma_{e q}<0 \\
\sigma_{e q}, \quad \text { if }\|\hat{\theta}\| \geq M_{\theta} \text { and } \sigma_{e q} \geq 0 \\
\sigma_{e q}=-\frac{\gamma s^{T} Y \hat{\theta}}{\|\hat{\theta}\|^{2}}
\end{array}\right.
$$


being $M_{\theta}$ the maximum possible value (assumed to be known) of the estimated deviation of the parameters in relation to their nominal values, and $\|\hat{\theta}\|^{2}$ the 2norm of $\hat{\theta}$. Let us now consider the dynamic model of Par2 (2). By neglecting $M_{t o t}$ (total mass of the robot, i.e. $M_{t o t}=M_{t p}+n \frac{M_{\text {forearm }}}{2}$ ), which would actually be represented by its estimate $\hat{M}_{t o t}$ in the implemented dynamic model of Par2 with the Dual Mode controller, the computation of heavy multiplications between the Jacobian matrices/derivative of Jacobian matrices are avoided. The dry friction is also neglected to avoid using the nonlinear sign(.) in the adaptation process. Therefore, a simplified dynamic model can be written (with the estimates of the system parameters $\hat{a}$, given below in (16)) in the form that follows:

$$
I_{t o t}(q, \hat{a}) \ddot{q}+G(q, \hat{a})+f(q, \dot{q}, \hat{a})=\tau
$$

being $G(q, \hat{a})$, in this case, given by:

$$
G(q, \hat{a})=-\frac{g}{2}\left(M_{\text {arm }}+M_{\text {forearm }}\right)\left[\begin{array}{l}
l_{1} \cos \left(q_{1}\right) \\
l_{2} \cos \left(q_{2}\right)
\end{array}\right]
$$

The regressor vector $Y=Y\left(q, \dot{q}, \dot{q}_{r}, \ddot{q}_{r}\right)$ can then be computed:

$$
I_{t o t}(q, \hat{a}) \ddot{q}_{r}+G(q, \hat{a})+f\left(q, \dot{q}_{r}, \hat{a}\right)=Y \hat{a}
$$

where $\dot{q}_{r}=\dot{q}_{d}+\lambda e_{j}$. Note that in (14) we use $\dot{q}_{r}$ and $\ddot{q}_{r}$ instead of $\dot{q}$ and $\ddot{q}$. The key idea comes from eliminating undesirable steady-state position errors by restricting them to evolve on a sliding surface [18] such as in robust sliding mode control [20].

By rewriting the simplified dynamics of the system such that it has the form given in (14), one can find that: 


$$
Y=\left[\begin{array}{cccc}
\ddot{q}_{1 r} & 0 & \dot{q}_{1 r} & -l_{1} \cos \left(q_{1}\right) \\
0 & \ddot{q}_{2 r} & \dot{q}_{2 r} & -l_{2} \cos \left(q_{2}\right)
\end{array}\right]
$$

and

$$
\hat{a}=\left[\begin{array}{c}
\hat{a}_{1} \\
\hat{a}_{2} \\
\hat{a}_{3} \\
\hat{a}_{4}
\end{array}\right]=\left[\begin{array}{c}
I_{\text {act }}+I_{\text {arm }} \\
I_{\text {act }}+I_{\text {arm }} \\
f_{v} \\
\frac{g}{2}\left(M_{\text {arm }}+M_{\text {forearm }}\right)
\end{array}\right]
$$

As the system does not have a sensor to measure the joint velocities, the velocity measurements are not available. So an estimator must be proposed and implemented (in order to calculate $Y, \hat{a}$ and $s$ in (6)). In the present work, three estimators have been proposed and experimentally compared. They are described in the sequel.

\section{PROPOSED OBSERVERS}

To deal with the unavailability of velocity measurements, three techniques have been implemented to estimate them. They are presented in the sequel:

\subsection{Lead-lab based observer}

The Lead-lag filter [21] has the following modified structure:

$$
G_{l l}(p)=\frac{p}{\tau_{1} p+1}
$$

where $p$ is the Laplace operator. So, the smaller $\tau_{1}$ is chosen, the more accurate the velocity estimation will be, but the more noise the estimated velocity will have. 
The idea of the Lead-lag based observer is then to approximately derivate the input signals (in our case, the joint positions), as shown in the diagram of Figure 6:

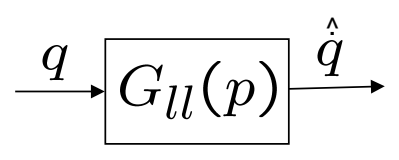

Figure 6: Block diagram of the Lead-lag based observer

This diagram is detailed as follows:

$$
\hat{\dot{q}}(t)=g_{l l}(t) * q(t)
$$

where $\dot{\hat{q}}(t)$ is the estimated joint velocity, $g_{l l}(t)$ is the inverse Laplace transform of $G_{l l}(p)$, i.e, $g_{l l}(t)=\mathcal{L}^{-1}\left[G_{l l}(p)\right]$ and the operator $*$ denotes the convolution between two functions.

The main advantages of this method are its simplicity and computational efficiency, but its main drawbacks are the following:

1. If $\tau_{1}$ is given a big value, a delay in the output will be generated and it will cause an inaccurate velocity estimation;

2. If $\tau_{1}$ is given a very small value, there may be a considerable amplification of the noise/quantization effects.

Then, $\tau_{1}$ must be chosen such that a compromise between 1 . and 2 . is achieved.

\subsection{Alpha-beta-gamma observer}

The Alpha-Beta (or Alpha-beta-gamma) observer [22] consists in a simplified form of Kalman Filter [23] for mechanical systems that have as their states the 
positions and the velocities. Its main idea consists in estimating the next step of the states of the system according to the usual equations of kinematics and to correct these estimations through the multiplication of the positions' estimation errors by constant gains. The structure of this observer is detailed as follows:

- Estimation of the next step's positions and velocities:

$$
\left\{\begin{array}{c}
x_{\text {prev }}(k+1)=x_{\text {innov }}(k)+T . v_{\text {innov }}(k)+\frac{1}{2} T^{2} \cdot a_{\text {innov }}(k) \\
v_{\text {prev }}(k+1)=v_{\text {innov }}(k)+T . a_{\text {innov }}(k)
\end{array}\right.
$$

where $x_{\text {prev }}(k+1)$ and $v_{\text {prev }}(k+1)$ are the estimated positions and velocities for the next step, $x_{\text {innov }}(k), v_{\text {innov }}(k)$ and $a_{\text {innov }}(k)$ are the updated positions, velocities and accelerations obtained through a correction based on the estimation error of the position, detailed as follows,

- Update of the estimated positions and velocities (also called innovation) based on the position measurement:

$$
\left\{\begin{array}{c}
x_{\text {innov }}(k)=x_{\text {prev }}(k)+\alpha \cdot\left(x_{\text {meas }}(k)-x_{\text {prev }}(k)\right) \\
v_{\text {innov }}(k)=v_{\text {prev }}(k)+\frac{\beta}{T} \cdot\left(x_{\text {meas }}(k)-x_{\text {prev }}(k)\right) \\
a_{\text {innov }}(k)=\frac{\gamma}{T^{2}} \cdot\left(x_{\text {meas }}(k)-x_{\text {prev }}(k)\right)
\end{array}\right.
$$

being $\alpha, \beta$ and $\gamma$ the update gains, $x_{\text {meas }}$ the measured positions and $T$ the sampling time.

In our case, the initial articular positions are known and the initial articular velocities are equal to zero, since the robot starts its movements from rest. So, $x_{\text {prev }}(1)$ and $v_{\text {prev }}(1)$ are initialized with these values, respectively. On the next step, based on the new measured articular positions, there is the innovation process, 
on which $x_{\text {innov }}, v_{\text {innow }}$ and $a_{\text {innov }}$ are calculated and then used to compute the new estimated states of the system $\left(x_{\text {prev }}\right.$ and $\left.v_{\text {prev }}\right)$ for the next step. To summarize, the basic principle of this observer is illustrated in the block diagram of Figure 7.

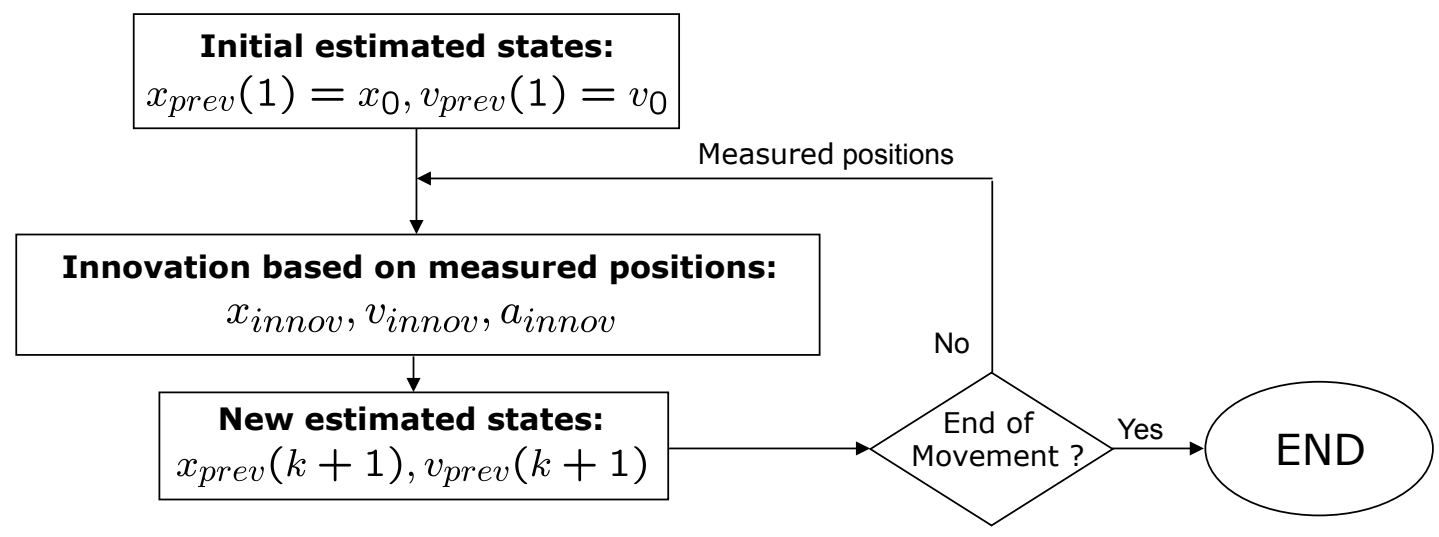

Figure 7: Basic principle of the Alpha-beta-gamma observer's algorithm

As the Lead-lag based observer, this observer has also the advantage of being very computationally efficient and simple to implement, but it can provide a better performance and robustness (cf. experimental results in section 6) since it is updated at each sample time. One of its main disadvantages is that its gains must be chosen such that a considerable amplification of the noises/quantization effect is avoided, but even with less aggressive gains, fairly decent performances can be achieved.

\subsection{High-gain observer}

The High-gain observer (HGO) is a model-based observer that was proposed in [24]. The main characteristic of this observer is that it takes into account the model of the system, so it can generate a very good performance if a precise model is available. However, even if this is not the case, this observer is robust because at each time step a correction of the estimated states is made according to the 
comparison between the estimated positions and the measured ones. Its description is given as follows:

$$
\left\{\begin{array}{c}
\dot{\hat{x}}_{1}=\hat{x}_{2}+\frac{1}{\epsilon} \alpha_{H G O 1}\left(x_{1}-\hat{x}_{1}\right) \\
\dot{\hat{x}}_{2}=\frac{1}{\epsilon^{2}} \alpha_{H G O 2}\left(x_{1}-\hat{x}_{1}\right)-\ddot{q} d+\hat{F}\left(\hat{x}, q_{d}, \dot{q}_{d}\right)+\hat{H}\left(\hat{x}_{1}, q_{d}\right) \tau^{s}
\end{array}\right.
$$

where

- $x_{1}$ represents the position error $\left(x_{1}=\tilde{q}\right)$ and $x_{2}$, its first derivative $\left(x_{2}=\dot{\tilde{q}}\right)$,

- $\hat{x}_{1}$ and $\hat{x}_{2}$ represent the estimated states,

- $\epsilon, \alpha_{H G O 1}$ and $\alpha_{H G O 2}$ are positive gains,

- $\hat{F}\left(\hat{x}, q_{d}, \dot{q}_{d}\right)=-I^{-1}\left(x_{1}, q_{d}\right)\left[C\left(x, q_{d}, \dot{q}_{d}\right)\left(x_{2}+\dot{q}_{d}\right)+G\left(x_{1}, q_{d}\right)\right]$, being the matrices $I, C$ and $G$ those of the dynamic model (1),

- $\hat{H}\left(\hat{x}_{1}, q_{d}\right)=I^{-1}\left(x_{1}, q_{d}\right)$,

- $\tau^{s}$ is the saturated torque (to avoid the 'peaking phenomena').

The main characteristics of such observer are:

1. a precise model of the system must be used in order to obtain good performances,

2. it is more complex (computationally and also concerning its implementation) than the two previously described observers.

In the sequel, the stability analysis of the Par2 robot under the control of the Dual Mode controller (complied with the High-gain observer for joint velocity estimation) will be presented. 


\section{STABILITY ANALYSIS}

For the stability analysis of the Par2 robot, the following proposition is made:

Proposition 1. The Par2 parallel manipulator, modeled by (2), subject to bounded disturbances $\left(\|d(t)\| \leq d_{\max }\right)$, in closed-loop with the Dual Mode nonlinear/adaptive controller (6) with adaptation law as in (8) (having $\hat{M}_{\text {tot }}$ and the dry friction neglected), and involving an estimated velocity with the High-gain observer is uniformly bounded under the following assumptions:

- $\|\hat{\theta}(0)\| \leq \theta_{M}$, therefore $\|\hat{\theta}(t)\| \leq \theta_{M}, \forall t>0$,

- $q_{d}, \dot{q}_{d}$ and $\ddot{q}_{d}$ are bounded (adequately chosen reference trajectories),

- the Jacobian and its inverse exist and are bounded by a known constant $\bar{J} \in \Re^{+}$ such that $\left\|J_{m}(\eta)\right\|,\left\|J_{m}^{-1}(\eta)\right\| \leq \bar{J}$. The minimum singular value of $J_{m}(\eta)$ is assumed to be greater than a known small positive constant $v>0$, such that $\operatorname{Max}\left\{\left\|J_{m}^{-1}(\eta)\right\|\right\}$ is known a priori, and hence, all kinematic singularities are avoided. The time-derivative of the Jacobian $\left(\dot{J}_{m}\right)$ is also assumed to be bounded. These assumptions are valid if one considers that the robot remains far from singularities [26].

Under these assumptions, the tracking error $e_{j}$ will exponentially converge to the residual domain given by:

$$
\left\|e_{j}\right\| \leq O\left(\frac{1}{\sqrt{\gamma}}\right)+O\left(\sqrt{\frac{\epsilon d_{\max } c(\bar{d})}{k}}\right)+O(\varepsilon)
$$

where $O($.$) represents the order of magnitude of the variables inside the brackets,$ $\gamma$ represents the adaptation gain, $\epsilon, d_{\max }$ and $c(\bar{d})$ correspond to configurable variables of the smooth variable structure term (where $\epsilon=\frac{1}{\alpha}$ (being $\alpha$ defined in the expression of Sat( $\alpha s), c f .(7)), d_{\max }$ is the upper bound of $\|d(t)\|$ and $\bar{d}>d_{\max }$ is a positive gain), $k$ represents a positive real constant which is considered proportional (by commodity) to the stabilizing term " $K$ " $\left(K=k I_{n \times n}\right)$ and $\varepsilon$ represents the gain of the High-gain observer.

Consider the complete dynamic model of Par2 (2). This complete dynamic model can be rewritten as:

$$
I_{t o t} \ddot{q}-M_{g} l \cos (q)+J_{m}^{T} M_{t o t} \ddot{X}+f_{v} \dot{q}=\tau
$$


where $M_{g}=\frac{M_{\text {arm }}+M_{\text {forearm }}}{2} g, \ddot{X}$ is the total Cartesian acceleration (having $\left[\begin{array}{ll}0 & g\end{array}\right]^{T}$ incorporated into it) and $l=l_{1}=l_{2}$. By considering that $\ddot{X}=J_{m} \ddot{q}+\dot{J}_{m} \dot{q}$, the final expression is obtained:

$$
I_{e q} \ddot{q}-M_{g} l \cos (q)+J_{m}^{T} M_{t o t} \dot{J}_{m} \dot{q}+f_{v} \dot{q}=\tau
$$

being $I_{e q}=I_{t o t}+J_{m}^{T} M_{t o t} J_{m}$. In [16], the system to be controlled is written in general form as:

$$
I(q, a) \ddot{q}+C(q, \dot{q}, a) \dot{q}+G(q, a)+f(q, \dot{q}, a)=\tau+d(t)
$$

where $a$ represents the real parameters of the system and $d(t)$ is a bounded disturbance. If one writes (24) in the general form of (25), we would have $I(q, a)=I_{e q}$, $C(q, \dot{q}, a)=J_{m}^{T} M_{t o t} \dot{J}_{m}, G(q, a)=-M_{g} l \cos (q), f(q, \dot{q}, a)=f_{v} \dot{q}$.

The idea of this analysis is to firstly consider that, initially, the adaptive term takes the complete dynamic model of Par2 into consideration, and then analyze the effect of the neglected $\hat{M}_{t o t}$ on the controlled system.

If the complete model of Par2 had been considered in the adaptive term, one would have the following control signal (which will be denoted as $\tau_{C M}$ instead of $\tau$ ):

$$
\tau_{C M}=\hat{I}_{e q} \ddot{q}_{r}+J_{m}^{T} \hat{M}_{t o t} \dot{J}_{m} \dot{q}_{r}-\hat{M}_{g} l \cos (q)+\hat{f}_{v} \dot{q}_{r}+\bar{d} S a t(\alpha s)+K s
$$

which can be rewritten as in (6):

$$
\tau_{C M}=Y \hat{a}+\bar{d} S a t(\alpha s)+K s
$$

being $Y \hat{a}=\hat{I}_{e q} \ddot{q}_{r}+J_{m}^{T} \hat{M}_{t o t} \dot{J}_{m} \dot{q}_{r}-\hat{M}_{g} l \cos (q)+\hat{f}_{v} \dot{q}_{r}$ in this case. By substituting this control law into (24), we obtain: 


$$
\begin{aligned}
I_{e q} \ddot{q}+J_{m}^{T} M_{t o t} \dot{J}_{m} \dot{q}-M_{g} l \cos (q)+f_{v} \dot{q}= & \hat{I}_{e q} \ddot{q}_{r}+J_{m}^{T} \hat{M}_{t o t} \dot{J}_{m} \dot{q}_{r}-\hat{M}_{g} l \cos (q)+ \\
& +\hat{f}_{v} \dot{q}_{r}+K s+\bar{d} \operatorname{Sat}(\alpha s)
\end{aligned}
$$

If one considers that $\ddot{q}=\ddot{q}_{r}-\dot{s}$ (cf. (5)), the following closed-loop expression is obtained:

$* * * * * * * * * * * *$ Right-hand side multiplied by $(-1)$

$$
\begin{aligned}
& \left(\hat{I}_{e q}-I_{e q}\right) \ddot{q}_{r}+\left(J_{m}^{T} \hat{M}_{t o t} \dot{J}_{m}-J_{m}^{T} M_{t o t} \dot{J}_{m}\right) \dot{q}_{r}-\left(\hat{M}_{g}-M_{g}\right) l \cos (q)+\left(\hat{f}_{v}-f_{v}\right) \dot{q}_{r}= \\
& -I_{e q} \dot{s}-J_{m}^{T} M_{t o t} \dot{J}_{m} s-K^{\prime} s-\bar{d} \operatorname{Sat}(\alpha s)
\end{aligned}
$$

where $K^{\prime}=\left(k+f_{v}\right) I_{n \times n}=k^{\prime} I_{n \times n}$, being $I_{n \times n}$ the identity matrix, used for commodity. As $f_{v}$ is a positive constant, the increase of $K^{\prime}$ in this analysis consequently means the increase of $K$. Therefore, $K^{\prime}$ will be considered as $K$ in the sequel, for a simplification of the notation. The expression above is equivalent to the closed-loop form:

$$
I(q, a) \dot{s}+C(q, \dot{q}, a) s+K s=Y \tilde{a}-\bar{d} S a t(\alpha s)+d(t)
$$

being $* * * Y \tilde{a}=-\left(\hat{I}_{e q}-I_{e q}\right) \ddot{q}_{r}-\left(J_{m}^{T}\left(\hat{M}_{t o t}-M_{t o t}\right) \dot{J}_{m}\right) \dot{q}_{r}+\left(\hat{M}_{g}-M_{g}\right) l \cos (q)-\left(\hat{f}_{v}-\right.$ $\left.f_{v}\right) \dot{q}_{r}{ }^{* * *}$. To prove the stability of the proposed algorithm, let us consider the following Lyapunov candidate function: 


$$
V(t)=\frac{1}{2}\left(s^{T} I(q, a) s+\tilde{a}^{T} \Gamma^{-1} \tilde{a}\right)
$$

being $\Gamma$ a symmetric positive definite matrix. The objective of the analysis is to have a positive definite $V(t)$ and analyze if $\dot{V}(t)$ is negative definite in all the state-space or if the region in which it would be negative can be made as large as one wants by adjusting the control gains. By computing the time-derivative of (31), we obtain:

$* * * * * * * * * * * * * * * * * * * *$

$$
\dot{V}(t)=s^{T} I(q, a) \dot{s}+\frac{1}{2} s^{T} \dot{I}(q, a) s+\tilde{a}^{T} \Gamma^{-1} \dot{\tilde{a}}
$$

Considering (30) and the skew-symmetry of $\frac{d I(q, a)}{d(t)}-2 C(q, \dot{q}, a)$, which ensures that the Coriolis term $C(q, \dot{q}, a)$ will be canceled in the sequel as $z^{T}\left(\frac{d I(q, a)}{d(t)}-2 C(q, \dot{q}, a)\right) z=$ 0 for any $z \in \Re$, we get:

$$
\dot{V}(t)=s^{T}(Y \tilde{a}+d(t)-\bar{d} S a t(\alpha s)-K s)+\tilde{a}^{T} \Gamma^{-1} \dot{\tilde{a}}
$$

$* * * * * * * * * * * * * * * * * * *$

By considering that the real parameters are constant, $\dot{\tilde{a}}=\dot{\hat{a}}=\dot{\tilde{\theta}}=\dot{\hat{\theta}}$. Therefore, with the definition of $\operatorname{Sat}(\alpha s)$ (cf. (7)), we get to:

$$
\dot{V}(t)=-s^{T} K s+s^{T} d(t)-s^{T} \frac{\bar{d}}{\|s\|+\epsilon} s+\tilde{\theta}^{T}\left(Y^{T} s+\Gamma^{-1} \dot{\hat{\theta}}\right)
$$

where $\epsilon=\frac{1}{\alpha}$. The proposed adaptation law $\left(\dot{\hat{\theta}}=-\Gamma \operatorname{Proj}\left(s^{T} Y, \hat{\theta}\right)\right)$ has the following properties [10]:

i) $\hat{\theta}$ is uniformly continuous, 
ii) if $\|\theta(0)\| \leq \theta_{M}$, then $\|\hat{\theta}(t)\| \leq \theta_{M}, \forall t>0$,

iii) $\|\operatorname{Proj}(y, \theta)\| \leq\|y\|$,

iv) $\tilde{\theta}^{T} \operatorname{Proj}(y, \hat{\theta}) \geq \tilde{\theta}^{T}$,

v) $\|\operatorname{Proj}(y, \hat{\theta})\|$ is bounded if $\|y\|$ is also bounded.

From (34) and property iv) of the projection algorithm (cf. (10),(11)), it is possible to affirm that:

$$
\begin{gathered}
\dot{V}(t) \leq-s^{T} K s+s^{T} d(t)-s^{T} \frac{\bar{d}}{\|s\|+\epsilon} s \\
\dot{V}(t) \leq-s^{T} K s+\|s\| d_{\max }-\bar{d} \frac{\|s\|^{2}}{\|s\|+\epsilon}
\end{gathered}
$$

As it is not possible to affirm that this function is negative definite, the properties of disturbance attenuation and of stability of the proposed system must be analyzed. For that, let us consider the two last terms of (36):

$$
f(\|s\|)=\|s\| d_{\max }-\bar{d} \frac{\|s\|^{2}}{\|s\|+\epsilon}
$$

This function can be evaluated through Figure 8. From (37), it is possible to notice that $f(\|s\|) \geq 0$ for $\|s\| \in\left[0, \frac{d_{\max } \epsilon}{\bar{d}-d_{\max }}\right]$, with $\bar{d}>d_{\max }$, and $f(\|s\|)<0$ for $\|s\|>\frac{d_{\max } \epsilon}{\bar{d}-d_{\max }}$. From Figure 8 , it is possible to notice that the interval in which the function is positive is as small as one wants by increasing the gain $\bar{d}$ and by decreasing the gain $\epsilon$ (configuration 1: $\bar{d}=220, \epsilon=0.3$; configuration 2: $\bar{d}=260$, $\epsilon=0.1$ and $d_{\max }=200$ in both cases), which guarantees that this function will be positive only for a small region of $\|s\|$ which will depend on $\bar{d}, \epsilon$ and $d_{\max }$.

By doing the derivative of $f(\|s\|)$ with respect to $\|s\|$ and making it equal to zero, the value of $\|s\|$ that maximizes $f(\|s\|)$ can be found, and is given by the 


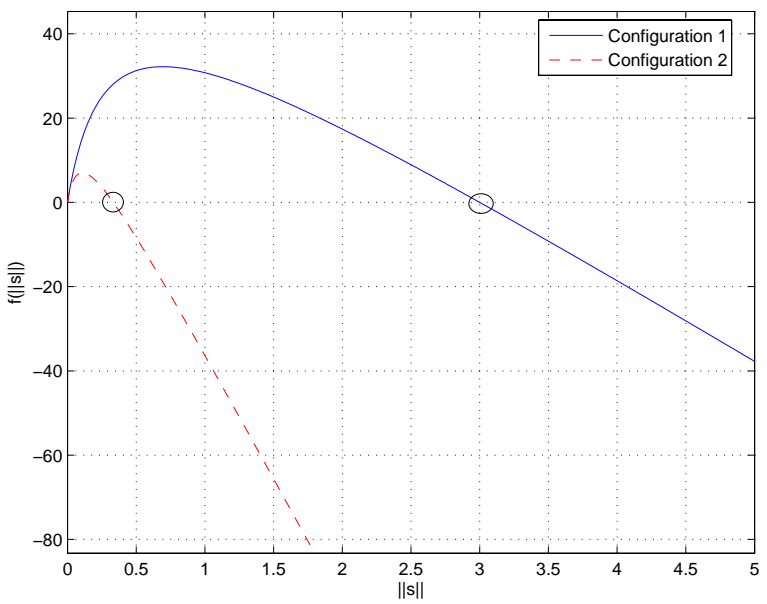

Figure 8: Effect of the increase of the gain $\bar{d}$ and decrease of the gain $\epsilon$ on the decrease of the positive region of $\|s\|$

following:

$$
\|s\|_{M}=-\epsilon+\epsilon \sqrt{\frac{\bar{d}}{\bar{d}-d_{\max }}}>0
$$

being the equivalent maximum value of $f(\|s\|)$ given by:

$$
f\left(\|s\|_{M}\right)=\epsilon d_{\max }\left[\left(-1+\sqrt{\frac{\bar{d}}{\bar{d}-d_{\max }}}\right)-\bar{d} \frac{\left(-1+\sqrt{\frac{\bar{d}}{\bar{d}-d_{\max }}}\right)^{2}}{d_{\max } \sqrt{\frac{\bar{d}}{\bar{d}-d_{\max }}}}\right]
$$

where the expression between the square brackets is denoted as $c(\bar{d})$, being then possible to rewrite (39) as:

$$
f\left(\|s\|_{M}\right)=\epsilon d_{\max } c(\bar{d})
$$

Then, an upper bound on $\mathrm{f}(\|s\|)$ can be the following:

$$
f(\|s\|) \leq f_{\max }=\epsilon d_{\max } c(\bar{d})
$$


where $f_{\max }$ can be reduced to arbitrarily small values by increasing $\bar{d}$ or decreasing $\epsilon$. We can therefore deduce that:

$$
\dot{V}(t) \leq-s^{T} K s+\epsilon d_{\max } c(\bar{d})
$$

It is now important to analyze the residual domain to which $s$ (and in consequence $e_{j}$ ) will converge. Let us firstly consider the property of non-singularity of inertia matrix:

$$
C_{m}\|s\|^{2} \leq s^{T} I(q, a) s \leq C_{M}\|s\|^{2}
$$

being $C_{m}=\lambda_{\min }(I(q, a))$ and $C_{M}=\lambda_{\max }(I(q, a))$ positive and constant scalars (equivalent to the minimum and maximum eigenvalues of $I(q, a)$, respectively), if the robot has revolute joints [27]. Adopting, by commodity, a matrix $K=k I_{n \times n}$, we have:

$$
\frac{k}{C_{M}} C_{m}\|s\|^{2} \leq \frac{k}{C_{M}} s^{T} I(q, a) s \leq k\|s\|^{2}=s^{T} K s
$$

If one considers (44) and (35), it is possible to conclude that:

$$
\dot{V}(t) \leq-\frac{k}{C_{M}} s^{T} I(q, a) s+\epsilon d_{\max } c(\bar{d})
$$

therefore, by substituting $s^{T} I(q, a) s=2 V(t)-\tilde{a}^{T} \Gamma^{-1} \tilde{a}$ (from (31)), we have:

$$
\dot{V}(t) \leq-\frac{2 k}{C_{M}} V(t)+\frac{k}{C_{M}} \tilde{a}^{T} \Gamma^{-1} \tilde{a}+\epsilon d_{\max } c(\bar{d})
$$

By choosing $\Gamma=\gamma P$, being $\gamma$ a positive real constant and $P$ a positive definite diagonal matrix, we can write: 


$$
\gamma^{-1} \frac{\|\tilde{\theta}\|^{2}}{\lambda_{M}(P)} \leq \tilde{\theta}^{T} \gamma^{-1} P^{-1} \tilde{\theta} \leq \gamma^{-1} \frac{\|\tilde{\theta}\|^{2}}{\lambda_{m}(P)}
$$

where $\lambda_{m}$ and $\lambda_{M}$ denote the minimum and the maximum eigenvalues of the matrix $\mathrm{P}$, respectively. By substituting (47) in (46) and considering that $\tilde{a}=\tilde{\theta}$, we obtain:

$$
\dot{V}(t) \leq-\frac{2 k}{C_{M}} V(t)+\frac{k\|\tilde{\theta}\|^{2}}{\gamma C_{M} \lambda_{m}(P)}+\epsilon d_{\max } c(\bar{d})
$$

The projection algorithm has the property that $\|\hat{\theta}\| \leq \theta_{M}$, which means that $\|\tilde{\theta}\| \leq 2 \theta_{M}$. Therefore, the following inequality is obtained:

$$
\dot{V}(t) \leq-\frac{2 k}{C_{M}} V(t)+\frac{4 k \theta_{M}^{2}}{\gamma C_{M} \lambda_{m}(P)}+\epsilon d_{\max } c(\bar{d})
$$

Let us consider $R=\frac{4 k \theta_{M}^{2}}{\gamma C_{M} \lambda_{m}(P)}+\epsilon d_{\max } c(\bar{d})$, then $(49)$ can be written as:

$$
\dot{V}(t) \leq-m V(t)+R
$$

where $m=\frac{2 k}{C_{M}}$. From the comparison lemma, it is possible to conclude that:

$$
V(t) \leq V(0) e^{-m t}+\frac{R}{m}\left(1-e^{-m t}\right)
$$

The following bound for $V(t)$ is then obtained:

$$
V(t) \leq V(0) e^{-m t}+\frac{2 \theta_{M}^{2}}{\gamma \lambda_{m}(P)}+\frac{\epsilon d_{\max } c(\bar{d})}{m}
$$

This means that the function $V(t)$ is upper bounded, being the error $s$ also bounded. It is now important to define which will be the bound of $s$. From (43) and (47), we can write: 


$$
\frac{C_{m}}{2}\|s\|^{2} \leq \frac{1}{2}\left(C_{m}\|s\|^{2}+\frac{\|\tilde{\theta}\|^{2}}{\gamma \lambda_{M}(P)}\right) \leq V(t)
$$

and

$$
V(0) \leq \frac{1}{2}\left(C_{M}\|s(0)\|^{2}+\frac{4 \theta_{M}^{2}}{\gamma \lambda_{m}(P)}\right)
$$

By substituting (53) and (54) in (52) and rearranging the different terms, we obtain:

$$
\|s\|^{2} \leq\left(\frac{C_{M}}{C_{m}}\|s(0)\|^{2}+\frac{4 \theta_{M}^{2}}{\gamma C_{m} \lambda_{m}(P)}\right) e^{-m t}+\frac{4 \theta_{M}^{2}}{\gamma C_{m} \lambda_{m}(P)}+\frac{2 \epsilon d_{\max } c(\bar{d})}{C_{m} m}
$$

Finally, substituting $m$ by its expression $\left(m=\frac{2 k}{C_{M}}\right)$ and applying the triangle inequality, we obtain:

$$
\|s\| \leq \sqrt{\frac{C_{M}}{C_{m}}}\|s(0)\| e^{-m t / 2}+\frac{2 \theta_{M}}{\sqrt{\gamma C_{m} \lambda_{m}(P)}}\left(1+e^{-m t / 2}\right)+\sqrt{\frac{C_{M} \epsilon d_{\max } c(\bar{d})}{C_{m} k}} \gamma \lambda_{m}(P)
$$

which can be rewritten as:

$$
\|s\| \leq C\|s(0)\| e^{-m t / 2}+\frac{k_{1}}{\sqrt{\gamma}}+C \sqrt{\frac{\epsilon d_{\max } c(\bar{d})}{k}}
$$

where $C=\sqrt{\frac{C_{M}}{C_{m}}}$ and $k_{1}=\frac{4 \theta_{M}}{\sqrt{C_{m} \lambda_{m}(P)}}$. The inequality (57) shows that the auxiliar error $s$ converges exponentially to an arbitrarily small region as the adaptation gain $\gamma$ and/or the parameter $\bar{d}$ are increased and/or if the constant $\epsilon$ is decreased.

Therefore, it was shown that the proposed Dual Mode controller, when applied to a manipulator modeled by (25) and considering the effects of disturbances uni- 
formly bounded by $\|d(t)\| \leq d_{\max }$, with initial condition of the estimated parameters satisfying $\|\hat{\theta}(0)\| \leq \theta_{M}$ and with $\bar{d}>d_{\max }$ guarantees the uniform boundedness of all the signals of the system in closed-loop. In this case, with no disturbance on the system $(d(t)=0)$, the auxiliar error $s$ will exponentially converge to the following residual domain:

$$
\|s\| \leq O\left(\frac{1}{\sqrt{\gamma}}\right)
$$

However, this conclusion would be valid only if the complete model had been taken into consideration in the adaptive term, being $d(t)=0$ in this case.

Now, let us consider the case of $\tau_{C M 2}$ which represents the two first terms of the left-hand side of (29). It is given by:

$\tau_{C M 2}=\left(\hat{I}_{a c t}-I_{a c t}+\hat{I}_{a r m}-I_{a r m}+J_{m}^{T} \hat{M}_{t o t} J_{m}-J_{m}^{T} M_{t o t} J_{m}\right) \ddot{q}_{r}+\left(J_{m}^{T} \hat{M}_{t o t} \dot{J}_{m}-J_{m}^{T} M_{t o t} \dot{J}_{m}\right) \dot{q}_{r}$

In the implemented case, $\hat{M}_{\text {tot }}$ was neglected such that the multiplication between Jacobian matrices and time-derivative of Jacobian matrices would not be necessary (as already mentioned in Section 3.2). Therefore, in the implemented case (cf. $(26),(27)$ ), one would have the following two first terms of $\tau_{C M}$, which will be named $\tau_{I C 2}$ instead of $\tau_{C M 2}$ :

$$
\tau_{I C 2}=\left(\hat{I}_{a c t}-I_{a c t}+\hat{I}_{a r m}-I_{a r m}-J_{m}^{T} M_{t o t} J_{m}\right) \ddot{q}_{r}+\left(-J_{m}^{T} M_{t o t} \dot{J}_{m}\right) \dot{q}_{r}
$$

It is then possible to notice that the difference between them is equal to:

$$
\tau_{C M 2}-\tau_{I C 2}=J_{m}^{T} \hat{M}_{t o t} J_{m} \ddot{q}_{r}+J_{m}^{T} \hat{M}_{t o t} \dot{J}_{m} \dot{q}_{r}
$$


Therefore, it would be considered as a disturbance to the controlled system:

$$
d(t)=\tau_{C M 2}-\tau_{I C 2}=J_{m}^{T} \hat{M}_{t o t} J_{m} \ddot{q}_{r}+J_{m}^{T} \hat{M}_{t o t} \dot{J}_{m} \dot{q}_{r}
$$

In [28], it is shown that the term $\bar{d} S a t(\alpha s)$ compensates for bounded disturbances if $\bar{d}>d_{\max }$. The disturbance in the present case must therefore be evaluated.

By considering that $s$ can be made as small as one wants by increasing $\gamma$ (while not considering saturation, which is the case of the present study), it is now necessary to show that the disturbance $d(t)$ is bounded. From (62) and (58), and considering the assumptions mentioned in the beginning of the present proposition, one can realize that:

- As the robot is assumed to remain far from singularities [26], $J_{m}$ and $\dot{J}_{m}$ are bounded,

- $\hat{M}_{\text {tot }}$ is bounded because of the adaptive law and the projection of the estimated parameters,

- $\dot{q}_{r}$ and $\ddot{q}_{r}$ are bounded, as $\dot{q}_{r}=\dot{q}_{d}-\lambda e_{j}$ and $\ddot{q}_{r}=\ddot{q}_{d}-\lambda \dot{e}_{j}$, being $\dot{q}_{d}$ and $\ddot{q}_{d}$ bounded, $\lambda$ a positive constant, and $e_{j}$ and $\dot{e}_{j}$ have been shown to be as small as one wants (while not at saturation) without disturbance. It is worth mentioning that $e_{j}(0)$ and $\dot{e}_{j}(0)$ are both equal to zero, which means that $e_{j}(t)$ and $\dot{e}_{j}(t)$ will represent bounded inputs for $\dot{q}_{r}$ and $\ddot{q}_{r}$ for $t>0$, because the increase of $\gamma$ will decrease $s$ (cf. (58)), and the smooth variable term will decrease the effect of the disturbance.

Therefore, it is possible to conclude that the auxiliary error will converge to the following residual domain: 


$$
\|s\| \leq O\left(\frac{1}{\sqrt{\gamma}}\right)+O\left(\sqrt{\frac{\epsilon d_{\max } c(\bar{d})}{k}}\right)
$$

This analysis shows that the proposed Dual Mode nonlinear/adaptive controller is able to guarantee the exponential convergence of the tracking errors of the Par2 parallel manipulator to the residual domain given by (63), which can be made as small as possible by increasing $\gamma$ and/or $\bar{d}$ and decreasing $\epsilon$ (considering that there is no saturation of actuation). However, in this analysis the whole state was considered available (both joint positions and velocities), which is not the case. As the joint velocities are not measured, they are estimated with the High-gain observer (HGO).

In [24], it was shown that the High-gain observer is able to recover the performance of a system under state feedback control for sufficiently small $\varepsilon$, and that it will act as a perturbation term of the order of $\|\zeta\|$, which is of the order of $\varepsilon$ to the closed-loop system under the state feedback control. Therefore, combining our stability analysis (presented above) and the result of [24] on the HGO, one can deduce that the closed loop system will converge to the following residual domain:

$$
\|s\| \leq O\left(\frac{1}{\sqrt{\gamma}}\right)+O\left(\sqrt{\frac{\epsilon d_{\max } c(\bar{d})}{k}}\right)+O(\varepsilon)
$$

As the tracking error $e_{j}$ is related to the auxiliar error $s$ by a linear filter, the property of exponential convergence of $s$ to a residual domain is preserved for $e_{j}$ and $\dot{e}_{j}$

\section{REAL-TIME EXPERIMENTAL RESULTS}

This section is divided in two main parts. The first part consists in presenting and discussing the real-time experimental results obtained by the application of the 
two proposed controllers complied with the High-gain observer, and the second part consists in presenting and discussing the real-time experimental results obtained by the application of the nonlinear Dual Mode controller complied with the three proposed observers, described in section 4, to the Par2 parallel manipulator described in section 1. In both cases, the platform of the robot has to go from some initial Cartesian position $\left(x_{d_{i}}, z_{d_{i}}\right)$ to the desired final Cartesian position $\left(x_{d_{f}}, z_{d_{f}}\right)$ and then return to the initial position $\left(x_{d_{i}}, z_{d_{i}}\right)$, concluding the cycle. $* * *$ The Par2 robot has two Wittenstein TPM 050 motors, which have a maximum torque of around 500 N.m and a gear ratio of 21. The angular position of each motor is obtained through a Heidenhain EQN1325 encoder that has a resolution of 8192 measurable positions per revolution [25]. The experiments were executed with a sampling time of $0.5 \mathrm{msec}$ (sampling frequency of $2 \mathrm{KHz}$ ) ***.

\subsection{Real-time experimental comparison between controllers}

The corresponding task space reference trajectory, as well as the illustration of the robot movements are plotted in Figure 9. The real-time experimental testbed is displayed in Figure 10, where:

- the PC used for the development of the control schemes is represented by item (1),

- the energy/drivers box, responsible for the control of Par2 parallel manipulator, is represented by item (2),

- the emergency stop button is represented by item (3),

- the Par2 parallel manipulator is represented by item (4). 

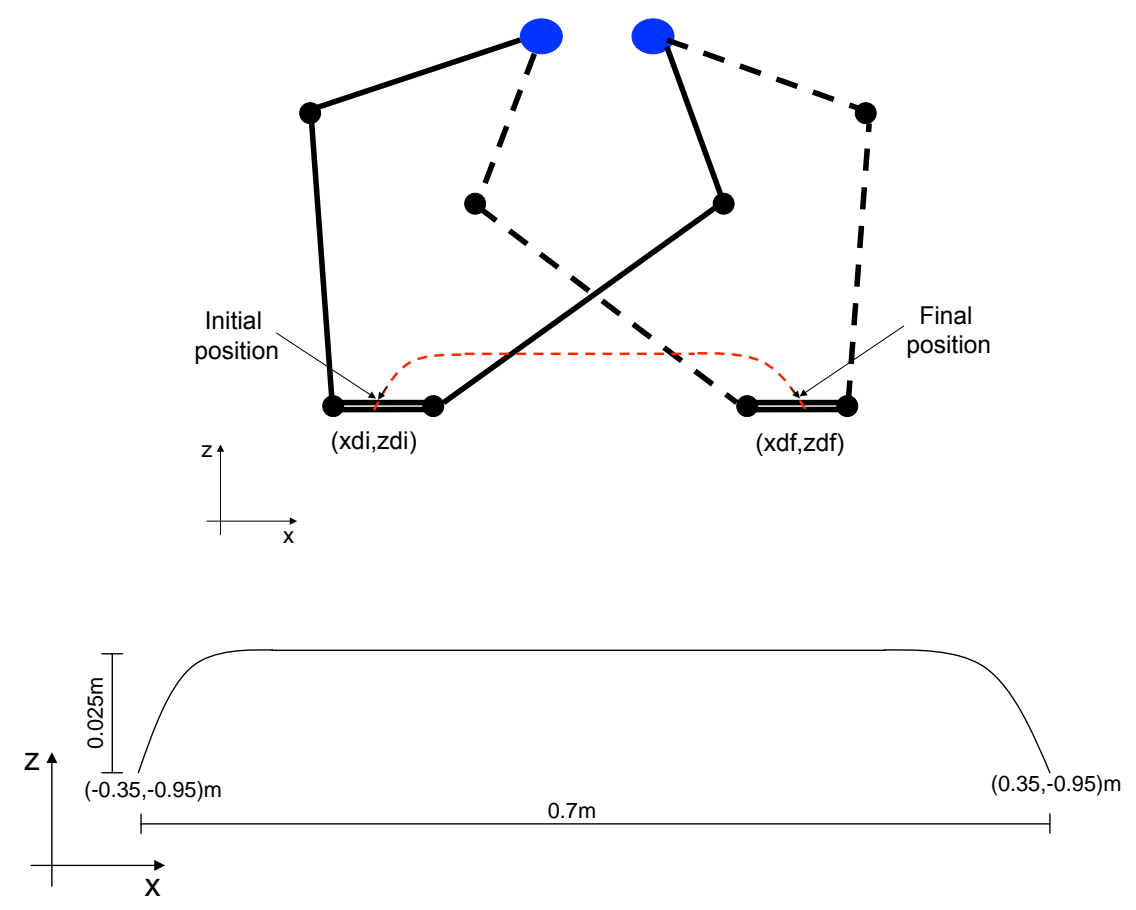

Figure 9: Illustration of the robot's movements (top) and the desired task space trajectory $x-z$ in a larger scale (bottom)

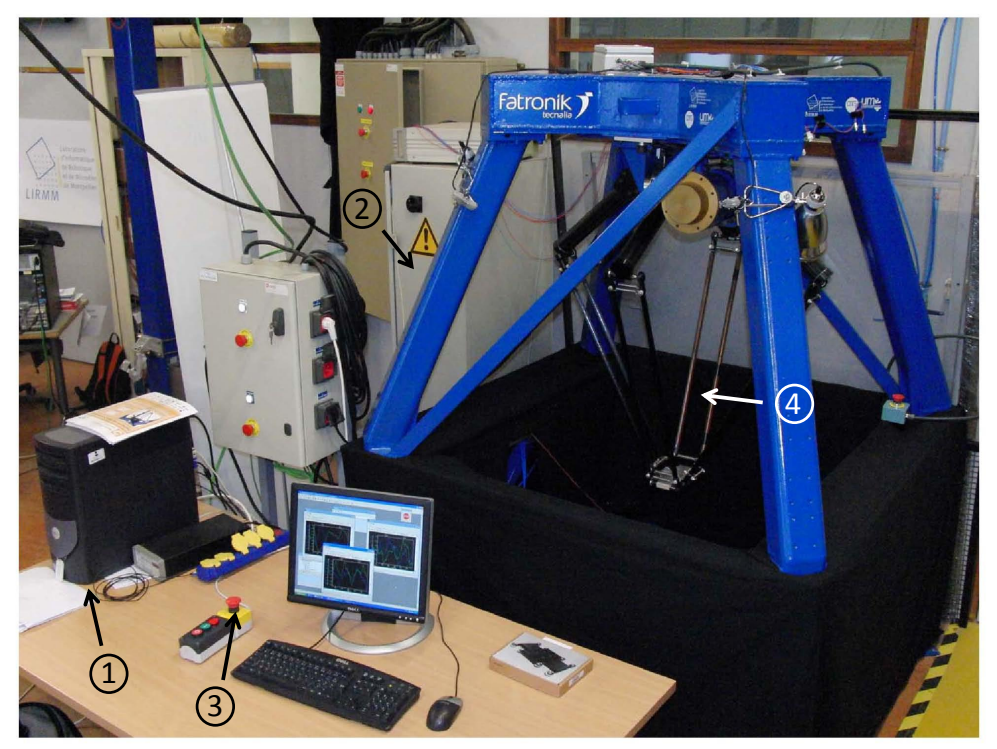

Figure 10: Par2 parallel manipulator experimental setup 
****** The trajectory tracking and the tracking errors in task space (computed from the forward kinematic model of the robot) obtained by the PD controller and by the DM controller for $20 \mathrm{G}$ of maximum acceleration are shown in Figures 11 and 12 and the control inputs are shown in Figure 13. The real-time implementation of both controllers consisted in 3 pick-and-place cycles. In such scenario, the main control objective is to obtain the best possible tracking precision, specially around the stop points (final positions of the trajectory, equivalent to the pick and the place positions), which are emphasized in Table 4. These positions occur for $t \in[0.125,0.175] s$ (final desired position) and for $t \in[0.325,0.375] s$ (back to the initial desired position), being repeated in the equivalent intervals of the subsequent cycles. The parameters of both PD and the proposed DM controllers (which were chosen experimentally until the best performances were achieved in each case) are summarized in Table 3.

Table 3: Parameters of the Control Approach

\begin{tabular}{|c|c|}
\hline \multicolumn{1}{|c||}{ Parameter } & Description \\
\hline$K_{p}=94.5$ & Proportional gain \\
\hline$K_{d}=2.1$ & Derivative gain \\
\hline$\lambda=25$ & Positive constant \\
\hline$K=2 I$ & Matrix gain \\
\hline $\bar{d}=2.5$ & Smooth variable structure gain \\
\hline$\alpha=0.05$ & Smooth variable structure slope \\
\hline$\epsilon=0.002, \alpha_{H G O 1}=\alpha_{H G O 2}=1$ & HGO gains \\
\hline$M_{\theta}=0.25$ & Maximum adaptative parameters' error \\
\hline$\gamma=0.3345$ & Adaptive gain \\
\hline$T_{s}=0.0005$ & Sampling time (s) \\
\hline$n=3$ & Number of cycles \\
\hline
\end{tabular}

The evolution of the control signals is shown in Figure 13, where it is possible to notice that the PD controller is delayed in comparison to the DM controller, 

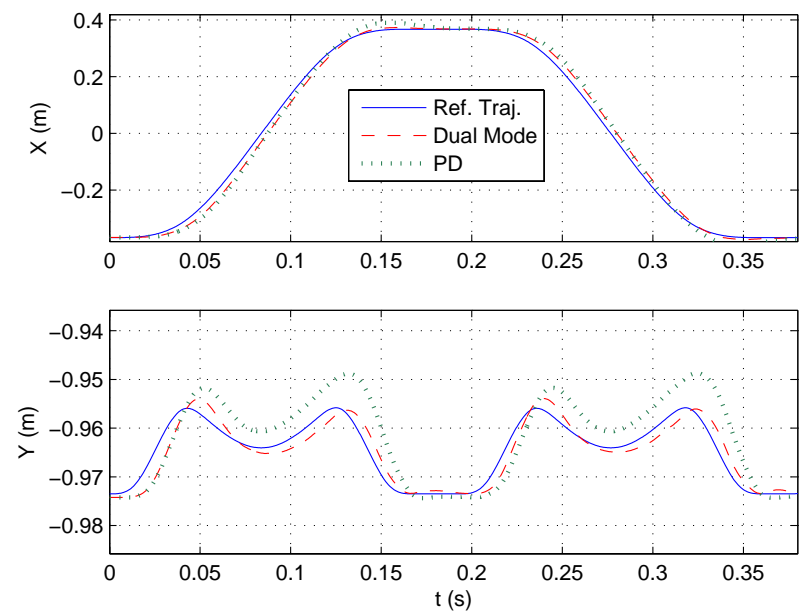

Figure 11: One cycle pick-and-place trajectory tracking (in task space) for 20G obtained with the PD controller (dashed line) and with the DM controller (dotted line)
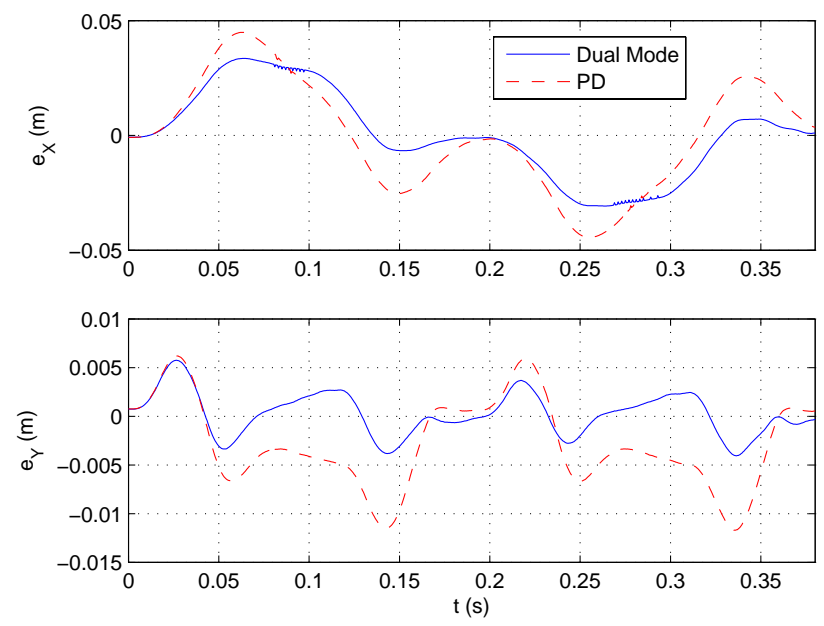

Figure 12: Tracking errors (in task space) for $20 \mathrm{G}$ obtained with the PD controller (dashed line) and with the DM controller (solid line) 


\subsection{Real-time comparison between state observers}

The trajectory tracking and tracking errors in task space obtained by the DM controller with the three proposed observers for $15 \mathrm{G}$ of maximum acceleration are shown in Figures 14-16. The velocity tracking is presented in Figure 17 and the estimated velocity errors are displayed in Figures 18 and 19. The control inputs are shown in Figures 20 and 21. The parameters of the proposed observers are presented in Table 5.

Table 5: Parameters of the proposed observers

\begin{tabular}{|c||c|}
\hline$\tau_{1}=0.001$ & LL time constant \\
\hline$\epsilon=0.002, \alpha_{H G O 1}=\alpha_{H G O 2}=1$ & HGO gains \\
\hline$\alpha_{a b g}=0.95, \beta_{a b g}=\gamma_{a b g}=0.5$ & ABG gains \\
\hline
\end{tabular}
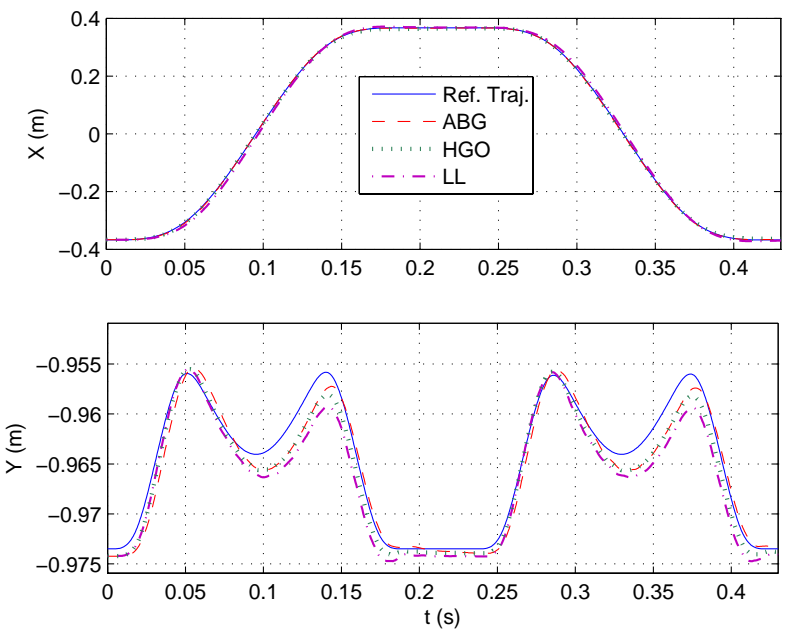

Figure 14: One cycle pick-and-place trajectory tracking (in task space) for 15G obtained by using the ABG (dashed line), the HGO (dotted line) and the LL (dash-dotted line) observers

The performance details of the three observers are summarized on Table 6 .

As for the main reasons why the Alpha-beta-gamma observer was able to gener- 


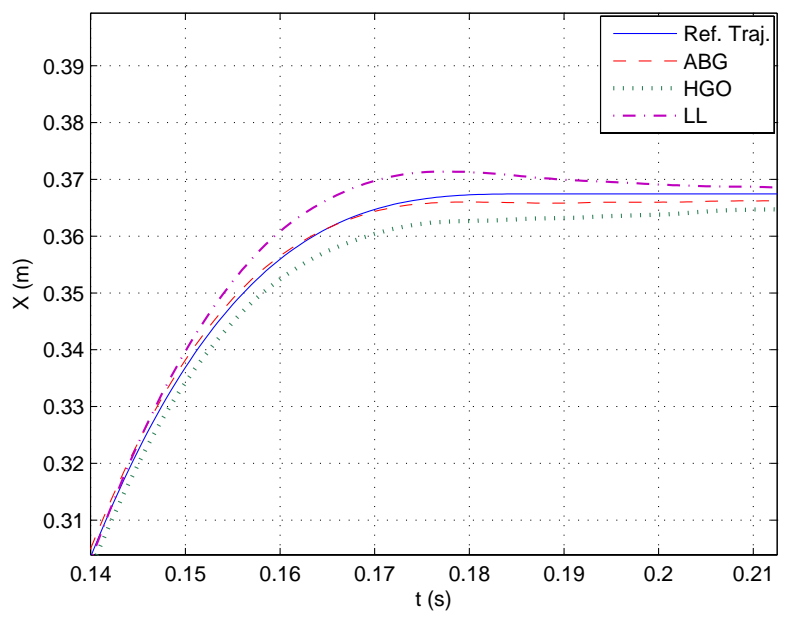

Figure 15: One cycle pick-and-place trajectory tracking (in task space) for $15 \mathrm{G}$ obtained by using the ABG (dashed line), the HGO (dotted line) and the LL (dash-dotted line) observers
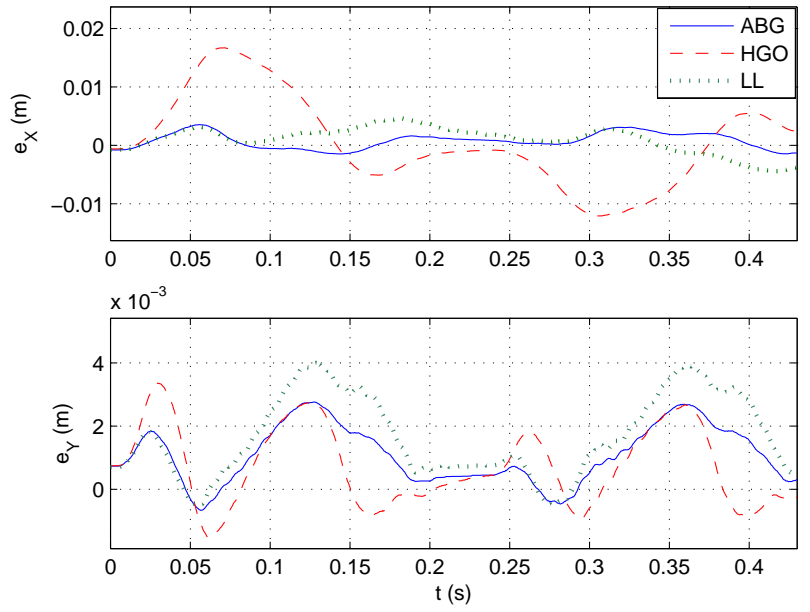

Figure 16: Tracking errors (in task space) for 15G obtained with the PD controller (dashed line) and with the DM controller (solid line) 

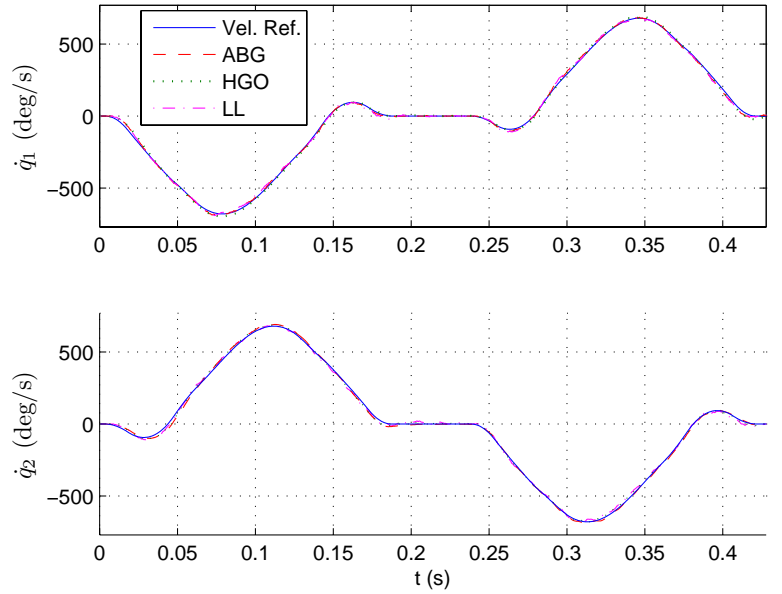

Figure 17: One cycle joint velocity tracking obtained by using the ABG (dashed line), the HGO (dotted line) and the LL (dash-dotted line) observers
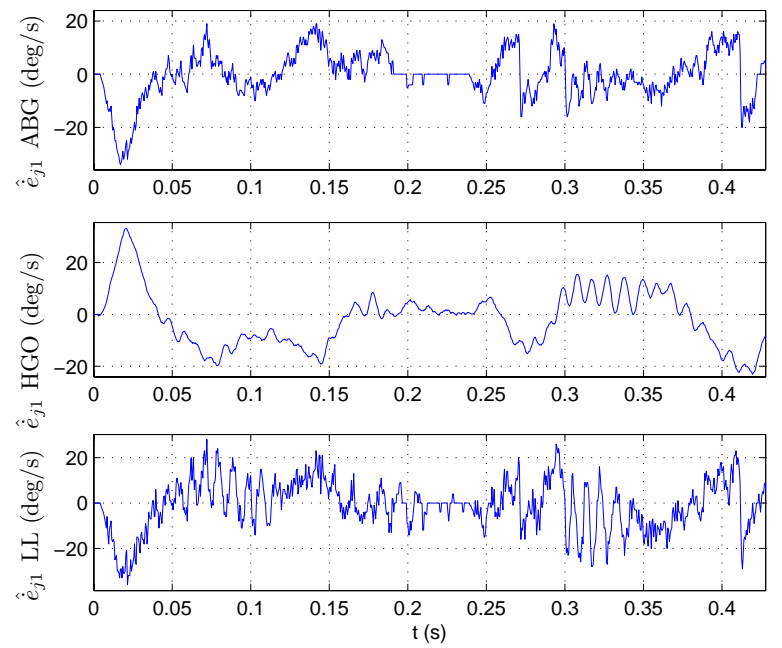

Figure 18: One cycle estimated joint velocity errors (peak errors inside limit of $5 \%$ of the velocity amplitudes) obtained by using each observer (Motor 1) 

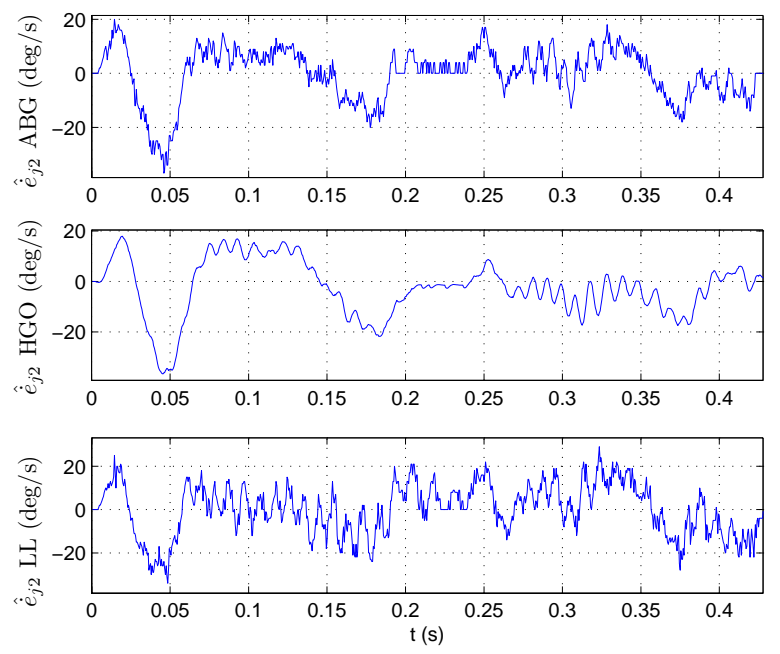

Figure 19: One cycle estimated joint velocity errors (peak errors inside limit of $5 \%$ of the velocity amplitudes) obtained by using each observer (Motor 2)
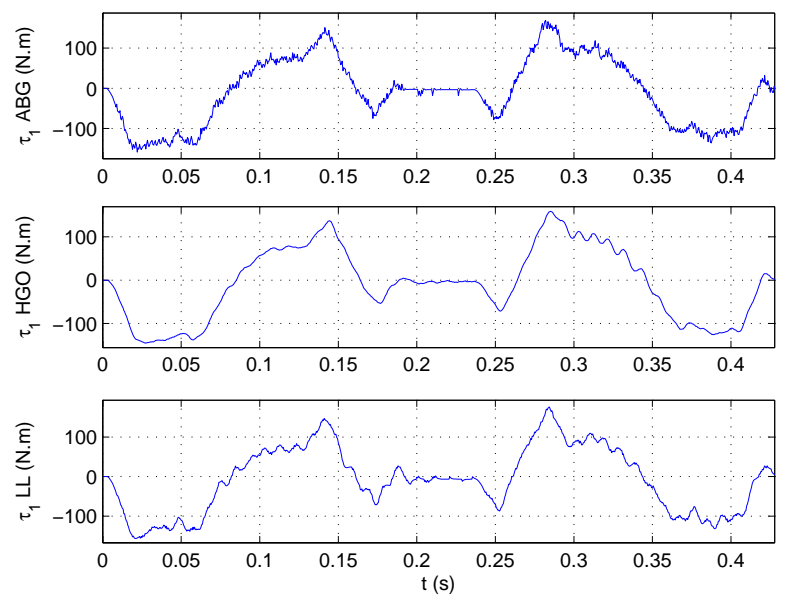

Figure 20: One cycle torques obtained by using each observer (Motor 1) 

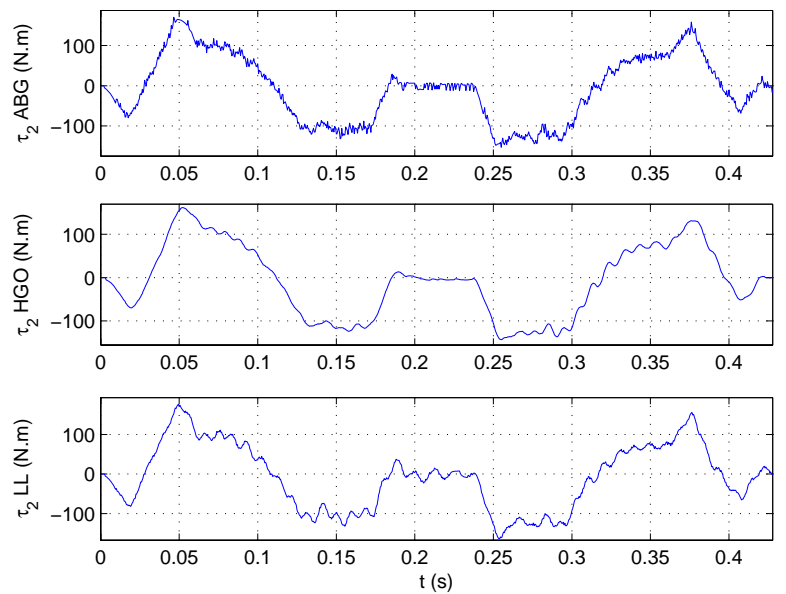

Figure 21: One cycle torques obtained by using each observer (Motor 2)

Table 6: Performance comparison between the observers used with the DM controller (15G)

\begin{tabular}{|c||c||c||c|}
\hline Performance & ABG & LL & HGO \\
\hline \hline Error peaks $(\mathrm{X})$ & {$[-1.47,3.56] \mathrm{mm}$} & {$[-4.39,4.63] \mathrm{mm}$} & {$[-12.07,16.71] \mathrm{mm}$} \\
\hline \hline Error peaks $(\mathrm{Y})$ & {$[-0.49,2.74] \mathrm{mm}$} & {$[-0.49,3.99] \mathrm{mm}$} & {$[-1.55,3.35] \mathrm{mm}$} \\
\hline \hline \multirow{2}{*}{ Control signals } & More noisy & More oscillating & Smoother \\
\cline { 2 - 4 } & \multicolumn{2}{|c|}{ Roughly similar amplitude values } \\
\hline
\end{tabular}

ate a better tracking performance than the Lead-lag based observer and the Highgain observer, one can mention that:

1. for the implementation of the High-gain observer, important simplifications on the model of the system were made such that it would be possible to represent it on the Lagrangian matrix form given in equation (1). As this observer is model-dependent, this may have caused a considerable loss of performance,

2. the Alpha-beta-gamma observer is naturally more performant than the Leadlag based observer because the latter consists only in a transfer function that will generate an approximate value of the velocity, while the $A B G$ observer 

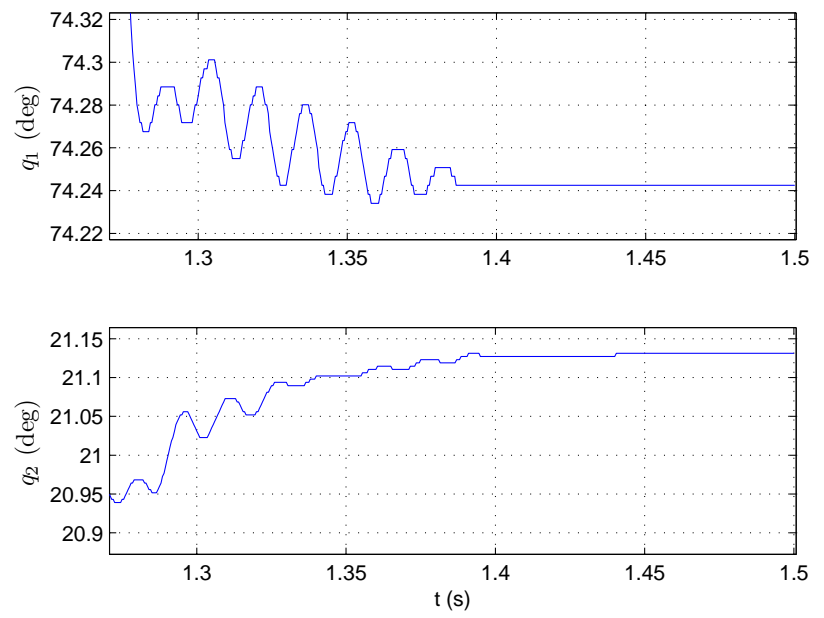

Figure 22: Zoom on the vibrations generated by the DM controller for $15 \mathrm{G}$ at the end of the third cycle

not only estimates the velocity but also corrects this estimation at each step according to the estimation error of the position.

\section{THE MECHANICAL VIBRATIONS ISSUE}

While increasing the acceleration of the robot (up to 20G), some vibrations could be noticed. By analyzing Figure 22 and 23, it is possible to notice the increase of the vibrations on the articular positions (in amplitude and in duration) caused by the increase of the acceleration from $15 \mathrm{G}$ to $20 \mathrm{G}$, which caused an amplification on the vibrations of the control signals (cf. Figures 24 and 25). Considering that our objective is to reach considerably higher accelerations, this issue will become very important.

In order to avoid such undesired behaviour of the system (loss of precision, or even damages to the mechanical structure of the robot) for higher accelerations, three solutions are suggested for future investigations, and they are summarized in 

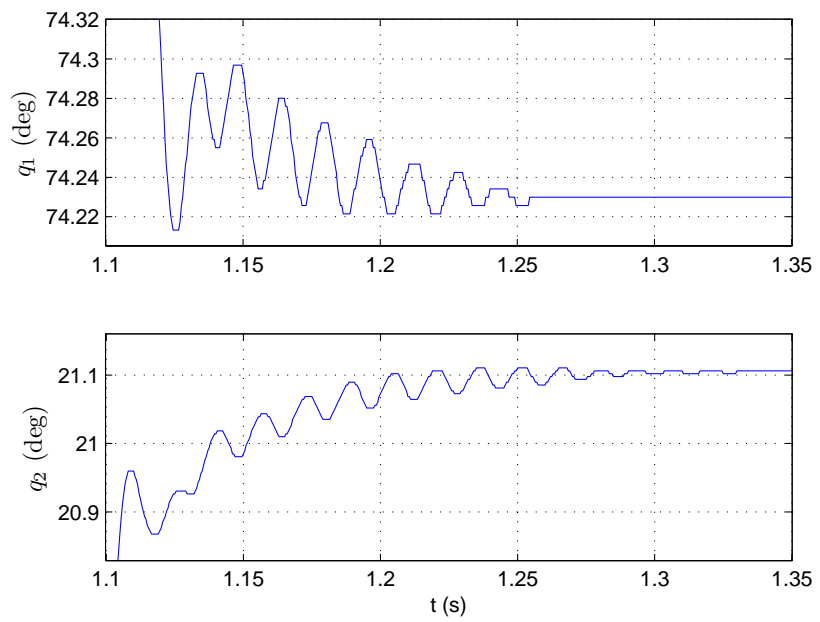

Figure 23: Zoom on the vibrations generated by the DM controller for $20 \mathrm{G}$ at the end of the third cycle
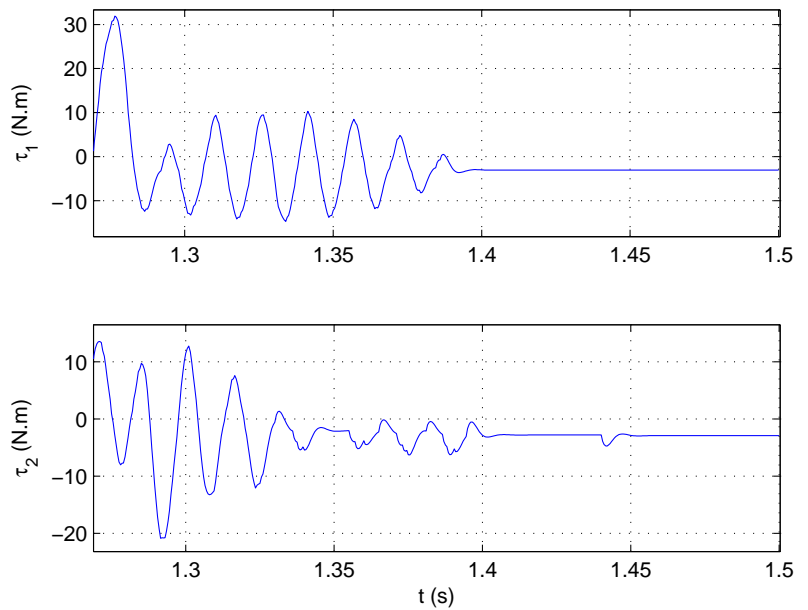

Figure 24: Zoom on the vibrations generated by the DM controller for $15 \mathrm{G}$ at the end of the third cycle 

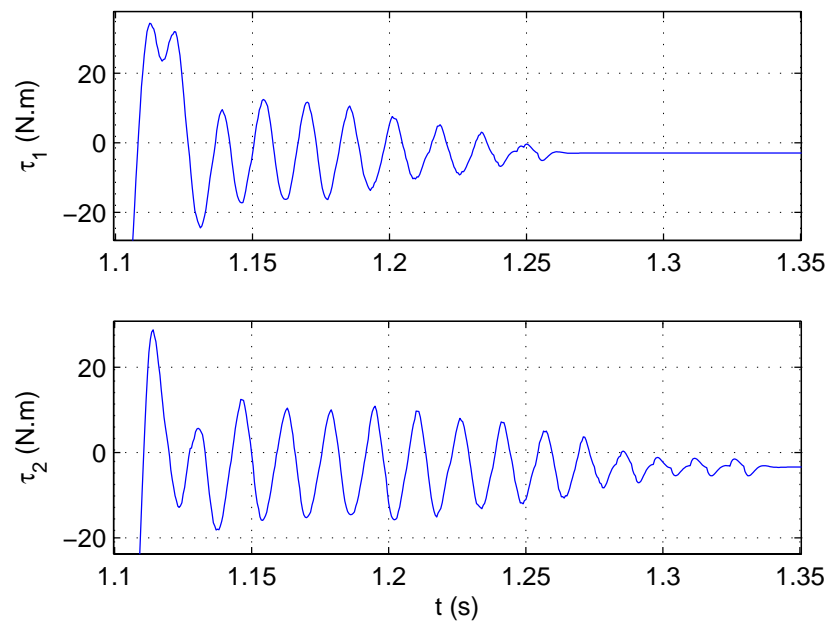

Figure 25: Zoom on the vibrations generated by the DM controller for $20 \mathrm{G}$ at the end of the third cycle

the following.

\subsection{Optimization of the reference trajectories}

This solution deals with the optimization of the reference trajectories with respect to some variables, such as maximum torques, maximum accelerations/decelerations, etc. In our case, the objective of the parametrization and the optimization of these parameters would be to minimize the arised mechanical vibration on the stop points, as illustrated in Figure 26.

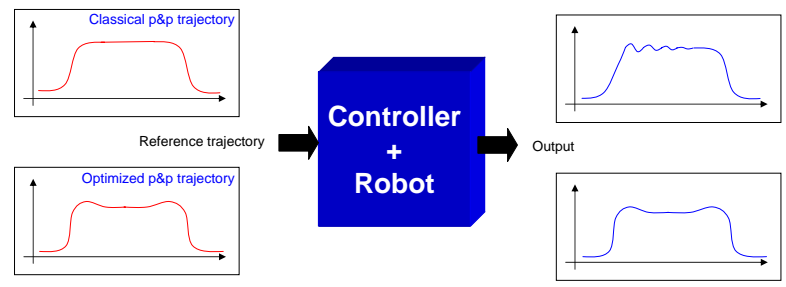

Figure 26: Effect of the pick-and-place (p\&p) reference trajectories' optimization on mechanical vibrations' reduction 

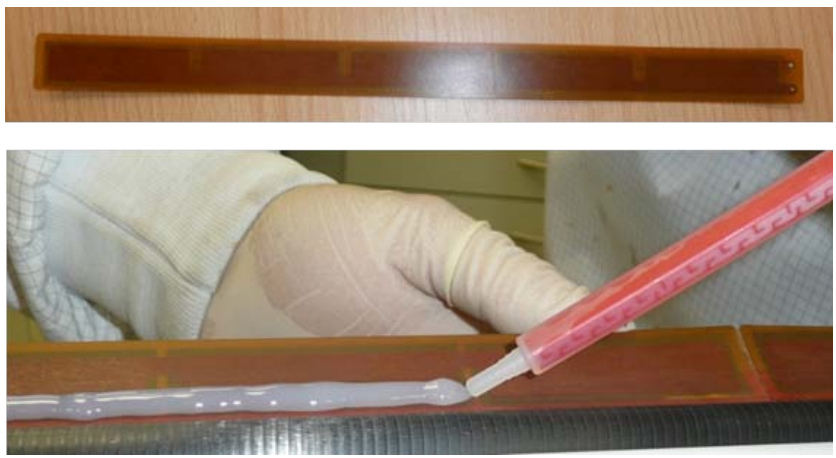

Figure 27: Piezo patch (top) and its fixation process (bottom)

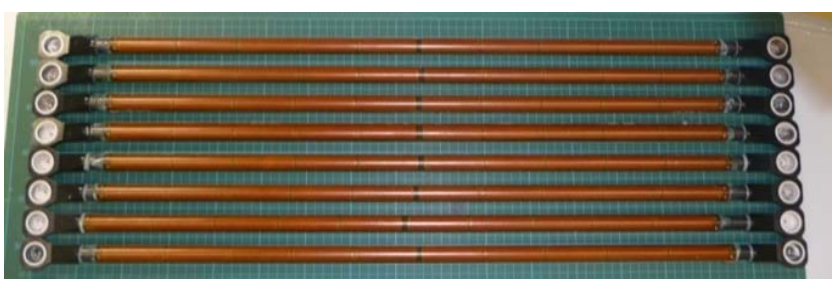

Figure 28: Arms of Par2 equipped with the piezo patches

\subsection{Utilization of piezoelectric actuators on the arms of the robot}

Controlled piezoelectric actuators can be used to damp/compensate vibrations. For that, the basic idea consists in generating adequate forces against arised vibrations. The first step of this solution is to attach piezoelectric actuators on the arms of the robot. Figure 27 shows the piezoelectric patches and how they are fixed (glued) on the arms. The arms of Par2 equipped with the piezoelectric patches are shown in Figure 28. The basic principle of sensing/actuation scheme used in [29] is presented in Figure 29, with $F(t)$ being the initial displacement field and $w(t)$ the white noise force disturbance. In the aim to test the feasibility of the piezoelectric actuators solution in our case, the experimental setup of Figure 30 was carried out.

The scheme used in [29] consisted in using piezo patches as sensors and also as actuators. In our tests, an accelerometer on the platform is used as a sensor instead. 


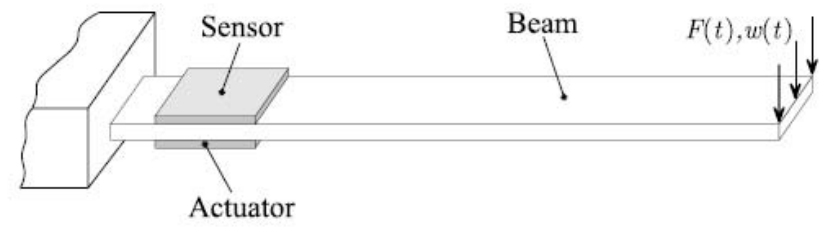

Figure 29: Diagram with the sensing/actuation scheme of the proposed solution in [29] to deal with vibrations

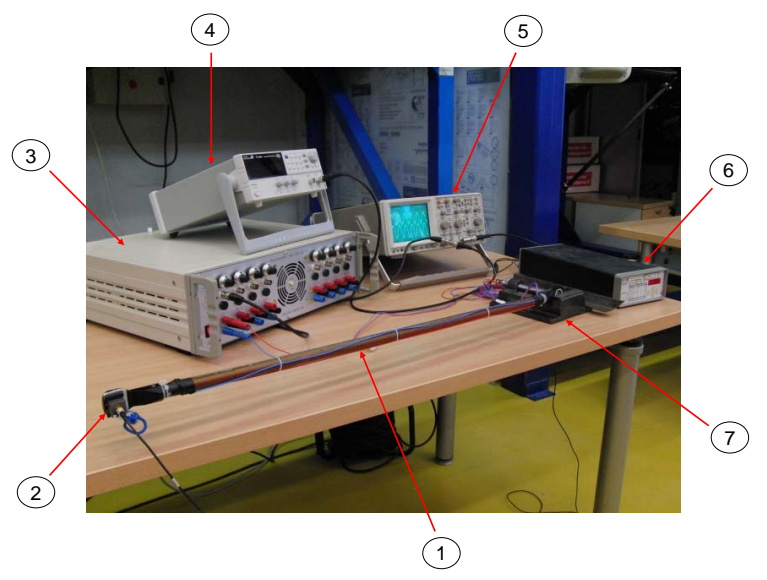

Figure 30: Experimental setup of the piezo-actuators 
In Figure 30, (1) is the beam (arm of the robot), (2) is the accelerometer, (3) is the amplifier (which has as an input signal between $-10 \mathrm{~V}$ and $10 \mathrm{~V}$, and an output signal between 0 and 400V), (4) is the low frequency signal generator, (5) is the oscilloscope, (6) is the signal conditioner for the accelerometer and (7) is the base where the beam is fixed.

*** In [30], an identification process was performed to obtain the nominal model of the patched beam that would synthesize a reduced order model with the $H_{\infty}$ loopshaping technique. Such approach was shown to be effective around the nominal operating point, but outside of such operating point, the saturation of the control efforts became relevant. In order to deal with such issue, an anti-windup strategy was employed, which allowed achieving vibration attenuation on the whole operation domain for a given configuration of the robot at the stop point.***

\subsection{Consideration of the arms' flexibility in the design of the controller}

During the design of the Dual Mode controller, the Par2 robot was considered rigid, which is not absolutely correct. The objective, when adopting this solution, is to take into account the vibrations in the dynamic model of the robot and to compensate them in the controller. For instance, in [31] a new method to control a flexible manipulator with noncollocated output was proposed and implemented in simulation, and a good control performance was obtained. The noncollocated output means that, instead of the joint velocity $\left(v_{a}\right)$, the end-point velocity $\left(v_{e}\right)$ is fedback to the controller through the link dynamics (cf. illustration in Figure 31). 


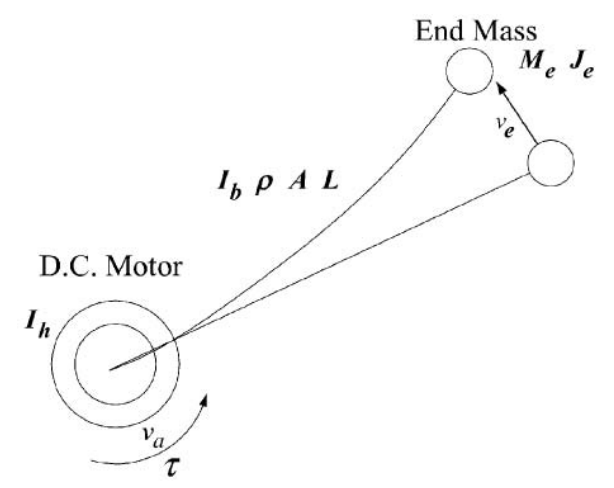

Figure 31: Diagram of the flexible arm used in [31]

\section{CONCLUSION AND FUTURE WORKS}

In this paper, a comparison between control/state estimation methods applied on the Par2 parallel manipulator was made, as well as a discussion about the mechanical vibrations issue that may become important when reaching very high accelerations. Real-time experiments were performed firstly to compare two controllers (a linear Proportional-Derivative (PD) and a nonlinear/adaptive Dual Mode (DM) controller) complied with the same High-gain observer (HGO) in order to estimate the articular velocities, and then to compare three state observers (a Lead-lag (LL) based, an Alpha-beta-gamma (ABG) and a High-gain observer) complied with the same nonlinear/adaptive DM controller. Results showed that the DM controller can generate a considerably better performance than the PD controller, and that the $\mathrm{ABG}$ observer was able to generate the best estimation of the joint velocities in this case study. Some small mechanical vibrations were noticed when reaching $20 \mathrm{G}$ of acceleration, which means that it can become an important issue for higher accelerations. Some suggestions have then been made for future investigations, in order to avoid/damp these vibrations. ${ }^{* * *}$ In the near future, an analysis about the 
effect of variation in the model parameters shall be made (such as experiments with different load conditions). In addition, experiments with higher involved accelerations shall be performed. New control schemes in the task (Cartesian) space shall also be proposed and implemented.***

\section{References}

[1] I. Bonev, The true origins of parallel robots, http://www.parallemic.org/Reviews/Review007.html, 2003.

[2] I. Bonev, Delta parallel robot - the story of success, http://www.parallemic.org/Reviews/Review002.html, 2001.

[3] M. Honegger, A. Codourey, E. Burdet, Adaptive control of the hexaglide, a 6-dof parallel manipulator, in: Proc. IEEE Conf. Robotics Automat., pp. 543$548,1997$.

[4] M. Honegger, R. Brega, G. Schweitzer, Application of a nonlinear adaptive controller to a 6-dof parallel manipulator, in: Proc. IEEE Conf. Robotics Automat., volume 2, pp. 1930-1935, 2000.

[5] J. J. Craig, Adaptive control of mechanical manipulators, Addison-Wesley Publishing Company, 1998.

[6] C.-C. Weng, W.-S. Yu, $H_{\text {inf }}$ Tracking Adaptive Fuzzy Integral Sliding Mode Control for Parallel Manipulators, in: Proc. IEEE World Congr. on Comp. Int., pp. 1-8, 2012.

[7] H. Abdellatif, B. Heimann, Advanced Model-Based Control of a 6-DOF Hexapod Robot: A Case Study, IEEE Trans. on Mech. 15, 269-279, 2010. 
[8] M. Diaz-Rodriguez, A. Valera, V. Mata, M. Valles, Model-Based Control of a 3-DOF Parallel Robot Based on Identified Relevant Parameters, IEEE Trans. on Mech. 18, 1737-1744, 02013.

[9] B. Xian, M. S. Queiroz, D. M. Dawson, M. L. McIntyre, A discontinuous output feedback controller and velocity observer for nonlinear mechanical systems, Automatica 40, 695-700, 2004.

[10] L. Hsu, R. Costa, B-mrac: Global exponential stability with a new model reference adaptive controller based on binary control theory, Contr. Th. and Adv. Tech. 10, 649-668, 1994.

[11] C. Baradat, V. Nabat, O. Company, S. Krut, F. Pierrot, Par2: a spatial mechanism for fast planar, 2-dof, pick-and-place applications, in: Proc. of the Second Int. Workshop on Fund. Issues and Fut. Res. Dir. for Par. Mech. and Manip., 2008.

[12] F. Pierrot, C. Baradat, V. Nabat, O. Company, S. Krut, M. Gouttefarde, Above $40 \mathrm{~g}$ acceleration for pick-and-place with a new 2-dof pkm, in: Proc. IEEE Conf. Robotics Automat., Kobe, Japan, 2009.

[13] R. Clavel, Delta, a fast robot with parallel geometry, in: International Symposium on Industrial Robots, pp. 91-100, 1988.

[14] V. Nabat, M. de la O Rodriguez, O. Company, S. Krut, F. Pierrot, Par4: Very high speed parallel robot for pick-and-place, in: Proc. IEEE/RSJ Int. Conf. Intel. Robots and Systems., pp. 553-558, 2005.

[15] L. Sciavicco, B. Siciliano, Modeling and control of robot manipulators, McGraw Hill, New York, 1996. 
[16] M. Spong, M. Vidyasagar, Robot dynamics and control, John Wiley \& Sons, New York, 1989.

[17] C. A. (part of Adept Technology Inc.), http://www.cerebellumautomation.com/addons.htm, 2009.

[18] J. Slotine, W. Li, Adaptive manipulator control: a case study, IEEE Trans. on Automat. Contr. 33 995-1003, 1988.

[19] J. Slotine, W. Li, Applied nonlinear control, Prentice Hall, 1991.

[20] S. K. S. C. Edwards, Sliding mode control: theory and applications, CRC Press, 1998.

[21] S. N. Norman, Control systems engineering, Wiley \& Sons, 4th Ed., 2004.

[22] R. Penoyer, The alpha-beta filter, C User's Journal 11, 73-86, 1993.

[23] R. E. Kalman, A new approach to linear filtering and prediction problems, Trans. of the ASME - Journal of Basic Eng. 35-45, 1960.

[24] K. W. Lee, H. K. Khalil, Adaptive output feedback control of robot manipulators using high-gain observer, Int. Journal of Control 67, 869-886, 1997.

[25] Wittenstein, http://www.servotechnica.ru/files/doc/documents/file-187.pdf.

[26] H. Cheng, Y. K. Yiu, Z. X. Li, Dynamics and control of redundantly actuated parallel manipulators, IEEE Trans. on Mechatronics 8, 483-491, 2003.

[27] M. Spong, Motion control of robot manipulators, Control handbook IEEE press, 1995. 
[28] F. A. Pazos, L. Hsu, Controle de robôs manipuladores em modo dual adaptativo/robusto, Revista Controle e Automação 14, 30-40, 2003.

[29] C. Vasques, J. D. Rodrigues, Active vibration control of smart piezoelectric beams: comparison of classical and optimal feedback control strategies, Computers \& Structures 84, 1402-1414, 2006.

[30] L. Douat, I. Queinnec, G. Garcia, M. Michelin, F. Pierrot, S. Tarbouriech, Identification and Vibration Attenuation for the Parallel Robot Par2, IEEE Trans. Contr. Syst. Tech. 22, 190-200, 2014.

[31] J.-H. Ryu, D.-S. Kwon, B. Hannaford, Control of a flexible manipulator with noncollocated feedback: Time-domain passivity approach, IEEE Trans. Robotics 20, 776-780, 2004. 